OPEN ACCESS

Edited by:

Attila Gacser,

University of Szeged, Hungary

Reviewed by:

István Pócsi,

University of Debrecen, Hungary

Remya Nair

Emory University, United States

*Correspondence:

Sneh Lata Panwar

sneh@mail.jnu.ac.in

Specialty section:

This article was submitted to Infectious Agents and Disease,

a section of the journal

Frontiers in Microbiology

Received: 14 October 2021 Accepted: 18 November 2021

Published: 06 January 2022

Citation

Husain F, Pathak P, Román E,

Pla J and Panwar SL (2022)

Adaptation to Endoplasmic Reticulum

Stress in Candida albicans Relies on the Activity of the Hog1

Mitogen-Activated Protein Kinase.

Front. Microbiol. 12:794855.

doi: 10.3389/fmicb.2021.794855

\section{Adaptation to Endoplasmic Reticulum Stress in Candida albicans Relies on the Activity of the Hog1 Mitogen-Activated Protein Kinase}

\author{
Farha Husain ${ }^{1}$, Prerna Pathak ${ }^{1}$, Elvira Román², Jesús Pla² and Sneh Lata Panwar ${ }^{1 *}$ \\ 1 Yeast Molecular Genetics Laboratory, School of Life Sciences, Jawaharlal Nehru University, New Delhi, India, \\ ${ }^{2}$ Departamento de Microbiología y Parasitología-IRYCIS, Facultad de Farmacia, Universidad Complutense de Madrid, \\ Madrid, Spain
}

Adaptation to ER stress is linked to the pathogenicity of $C$. albicans. The fungus responds to ER stress primarily by activating the conserved Ire1-Hac1-dependent unfolded protein response (UPR) pathway. Subsequently, when ER homeostasis is re-established, the UPR is attenuated in a timely manner, a facet that is unexplored in $C$. albicans. Here, we show that $C$. albicans licenses the HOG (high-osmolarity glycerol) MAPK pathway for abating ER stress as evidenced by activation and translocation of Hog1 to the nucleus during tunicamycin-induced ER stress. We find that, once activated, Hog1 attenuates the activity of Ire1-dependent UPR, thus facilitating adaptation to ER stress. We use the previously established assay, where the disappearance of the UPR-induced spliced HAC1 mRNA correlates with the re-establishment of ER homeostasis, to investigate attenuation of the UPR in C. albicans. hog1 $\Delta / \Delta$ cells retain spliced HAC1 mRNA levels for longer duration reflecting the delay in attenuating Ire1-dependent UPR. Conversely, compromising the expression of Ire1 (ire1 DX mutant strain) results in diminished levels of phosphorylated Hog1, restating the cross-talk between Ire1 and HOG pathways. Phosphorylation signal to Hog1 MAP kinase is relayed through Ssk1 in response to ER stress as inactivation of Ssk1 abrogates Hog1 phosphorylation in C. albicans. Additionally, Hog1 depends on its cytosolic as well as nuclear activity for mediating ER stress-specific responses in the fungus. Our results show that HOG pathway serves as a point of cross-talk with the UPR pathway, thus extending the role of this signaling pathway in promoting adaptation to ER stress in C. albicans. Additionally, this study integrates this MAPK pathway into the little known frame of ER stress adaptation pathways in C. albicans.

Keywords: Candida, tunicamycin, endoplasmic reticulum stress, unfolded protein response, HOG MAPK, Ire1, Hac1

\section{INTRODUCTION}

The endoplasmic reticulum (ER), in all eukaryotes, facilitates the efficient folding and transport of secretory and membrane proteins to their targeted locations (Mori et al., 1993). Protein biosynthesis in the ER is guided by chaperones and folding enzymes in a fail-safe manner by quality control systems. However, the increased demand for secretion during environmental or developmental 
changes overwhelms the protein folding machinery within the ER. Consequentially, the probability of unwarranted interactions during protein folding is enhanced, resulting in the accumulation of misfolded proteins in this organelle. This imbalance in the protein-folding homeostasis, referred to as ER stress, activates adaptive signaling pathways to alleviate the stress. The evolutionary conserved unfolded protein response (UPR) pathway plays a key role in adapting to ER stress in Saccharomyces cerevisiae and pathogenic fungi (Krishnan and Askew, 2014).

Pathogenic fungi including C. albicans encounter ER stress during infection in a mammalian host. C. albicans, as a harmless commensal, resides in the gastrointestinal tract (GI), mouth, skin, and female reproductive tract of healthy individuals (Kennedy and Volz, 1985; Achkar and Fries, 2010; Ghannoum et al., 2010; Kumamoto, 2011; Calderone and Clancy, 2012; Kan et al., 2020). The benign commensal turns into a pathogen, causing lifethreatening invasive and systemic candidiasis, when the host develops immune deficiency, epithelial damage, or microbiota dysbiosis (Edmond et al., 1999; Perlroth et al., 2007; Mayer et al., 2013; Bongomin et al., 2017). For successful infection, this fungus activates the ER stress response pathways to strike a balance between adaptation to ER stress and ER stress-induced cell death. The ER stress-induced UPR pathway in C. albicans is comprised of the conserved ER-resident transmembrane protein kinase Ire1 and its downstream transcription factor Hac1 (Sircaik et al., 2021). In S. cerevisiae, binding of misfolded proteins to the ER lumenal domain of Ire1 triggers its autophosphorylation and oligomerization, and activates ribonuclease (RNase) activity. Ire1 through its RNase activity excises the 252-bp and 19-bp intron from S. cerevisiae and C. albicans HAC1 mRNA, respectively, thus generating Hacl protein (Cox et al., 1993; Kohno et al., 1993; Wimalasena et al., 2008; Korennykh et al., 2009; Sircaik et al., 2021). Thereafter, Hacl coordinates the restoration of ER homeostasis by initiating the expression of proteins involved in protein folding and secretion and regulating the disposal of misfolded proteins by ER-associated degradation (ERAD) (Wimalasena et al., 2008; Ruggiano et al., 2014; Chang et al., 2018). In yeast, once protein-folding homeostasis is re-established, the Ire1-dependent UPR activity terminates, signaling recovery from the stress (Chawla et al., 2011; Chang et al., 2018). Sustained expression of the ER stress-induced spliced HAC1 mRNA and overexpression of Ire1 results in cell death in S. cerevisiae, reflecting the importance of timely termination of the UPR (Kawahara et al., 1997).

In C. albicans, while events leading to the activation of the Ire1-dependent UPR have been established (Sircaik et al., 2021), steps that mediate its timely attenuation remain uninvestigated. High-osmolarity glycerol (HOG) MAP kinase pathway promotes recovery from ER stress by mediating attenuation of the Ire1dependent UPR and regulating the expression of a specific subset of genes (HSP12, YMR103C, and YPLO88W) during late stage of ER stress response in $S$. cerevisiae. Induction of autophagy, considered as one of the downstream events during ER stress in yeast, is also regulated by Hog1 in the budding yeast. Hog1, by regulating the stability of Atg8, a critical autophagy protein, contributes to the activation of autophagy. Therefore, Hog1 activation facilitates modulation of multiple cellular processes specifically during the late stage of ER stress, resulting in counteracting the stress in S. cerevisiae (Bicknell et al., 2010). In C. albicans, Hog1 is activated by phosphorylation in response to osmotic and oxidative stresses (Alonso-Monge et al., 2003; Smith et al., 2004), regulates virulence traits (Munro et al., 2007; Su et al., 2013; Hopke et al., 2016), and is crucial for the survival of the fungus within the host (Alonso-Monge et al., 1999; Arana et al., 2007; Prieto et al., 2014). Subsequent to osmostress-induced phosphorylation, Hog1 translocates to the nucleus (Arana et al., 2005; Kaloriti et al., 2014) where it activates the transcriptional program that promotes adaptation to various stresses (Enjalbert et al., 2006). Dephosphorylated Hog1 migrates back to the cytoplasm where it is required for maintaining signal fidelity and impeding cross-talk to other MAPK pathways (Arana et al., 2005; Eisman et al., 2006; Cheetham et al., 2011). Despite the central role of Hogl in handling multiple stresses, its role during ER stress remains unaddressed in C. albicans.

Here, we explore the role of $C$. albicans Hog1 and bring forward the mode of action of this MAP kinase during tunicamycin-induced ER stress. We show that Hog1 is phosphorylated in Ssk1-dependent manner and migrates to the nucleus specifically during the late stage of ER stress response. For mediating ER stress resistance, Hog1 is dependent not only on its nuclear activity but also requires its cytosolic activity, thus highlighting the role of Hog1-dependent cytosolic proteins during ER stress in C. albicans. Moreover, once activated, HOG MAPK pathway promotes adaptation to ER stress by facilitating the timely attenuation of the UPR in C. albicans. All told, the finding that HOG pathway abates ER stress by orchestrating the Ire1-dependent UPR uncovers a new role for this MAP kinase in the pathogenic fungus.

\section{MATERIALS AND METHODS}

\section{Strain, Chemicals, and Growth Conditions}

The C. albicans strains used in this study are listed in Table $\mathbf{1 .}$ All strains were maintained as frozen stocks and propagated at $30^{\circ} \mathrm{C}$ in defined medium, YEPD (1\% yeast extract, $2 \%$ peptone, and $2 \%$ glucose). To stimulate the UPR in C. albicans, stationary phase cells were grown to mid-exponential phase in YEPD at $30^{\circ} \mathrm{C}$ with shaking $(220 \mathrm{rpm})$, containing tunicamycin at indicated concentration. All the supplements and chemicals, $\beta$-mercaptoethanol, tunicamycin, and Calcofluor white, were purchased from Sigma-Aldrich. Dithiothreitol was obtained from SRL (Sisco Research Laboratories Pvt. Ltd).

\section{Strain Construction}

A transient CRISPR system to target SSK1 was used that consisted of separate CaCAS9 and sgRNA expression cassettes. CaCAS9 cassette was amplified by PCR from pV1093 with the pair of primers CaCas9/for and CaCas9/rev. We chose a gRNA 219 bp downstream from the SSK1 start codon and the sgRNA was generated through three PCRs steps to fuse SNR52 promoter (amplified in PCR1 with the primers SNR52/F and SNR/R_SSK1) and the sgRNA (amplified in PCR2 with primers 
TABLE 1 | Strains used in this study.

\begin{tabular}{|c|c|c|c|}
\hline Parent & Genotype & Strain & Source of reference \\
\hline SC5314 & & Wild type & Gillum et al., 1984 \\
\hline RM1000 & $\begin{array}{l}\text { Ura3 } \Delta: \text { imm434/ura3D } \Delta: i m m 434 \\
\text { his1 } 1: \text { hisG/his1 } 1: \text { hisG }\end{array}$ & Wild type & Negredo et al., 1997 \\
\hline CNC15 & $\begin{array}{l}\text { RM1000 } \\
\text { hog1:hisG/hog1:hisG }\end{array}$ & $\operatorname{hog} 1 \Delta / \Delta$ & Arana et al., 2005 \\
\hline BRD4 & $\begin{array}{l}\text { RM1000 } \\
\text { pbs2 } 2: \text { cat/pbs2 } 2: \text { cat }\end{array}$ & $p b s 2 \Delta / \Delta$ & Arana et al., 2005 \\
\hline BRD8 & $\begin{array}{l}\text { RM1000 } \\
\text { hog1:hisG/hog1:hisG pbs2 } \Delta: \text { cat/pbs } 2 \Delta \\
\text { :cat }\end{array}$ & hog1pbs2 $\Delta / \Delta$ & Arana et al., 2005 \\
\hline hAHGl & $\begin{array}{l}\text { RM1000 } \\
\text { hog1:hisG/hog1:hisG ACT1p-HOG1 GFP:leu2/LEU2 }\end{array}$ & HOG1-GFP & Arana et al., 2005 \\
\hline BRD4G1 & pbs2 $\Delta: c a t / p b s 2 \Delta: c a t A C T 1 p-P B S 2-G F P: l e u 2 / L E U 2$ & $p b s 2 \Delta / \Delta-P B S 2$ & Arana et al., 2005 \\
\hline CSSK21 & ura3:imm434/ura3:imm434 ssk1:hisG/ssk1:hisG-URA3-hisG & $\operatorname{ssk} 1 \Delta / \Delta$ & Arana et al., 2005 \\
\hline REP3 & ura3:imm434/ura3:imm434 his1:hisG/his1:hisG sho1:hisG/sho1:hisG-URA3-hisG & $\operatorname{sho1} \Delta / \Delta$ & Arana et al., 2005 \\
\hline CW906 & $\begin{array}{l}\text { ura3 } \Delta \text { iro 1 } \Delta: \lambda \text { imm434arg4:hisG, his 1:hisG ARG4:IRE1-DX1 } \\
\text { ura3 } \Delta \text { iro1 } \Delta: \lambda \text { imm434arg4:hisGhis1:hisG } \Delta \text { ire1:URA3 }\end{array}$ & ire1 DX & Woolford et al., 2016 \\
\hline TW04 & ura3:kimm434/ura3:kimm434,his1:hisG/h is 1:hisG, arg4:hisG/arg4:hisG, hac1:loxP/hac1:loxP,Clp30 & hac1 $\Delta / \Delta$ & $\begin{array}{l}\text { Wimalasena et al., } \\
\qquad 2008\end{array}$ \\
\hline $\mathrm{FH} 1$ & $\begin{array}{l}\text { ura3 } \Delta \text { iro1 } \Delta: \lambda \text { imm434arg4:hisG, his1:hisG ARG4:IRE-DX1 ura3 } \Delta \text { iro1 } \Delta: \lambda \text { imm434arg4:hisGhis1:hisG } \\
\text { sire1:URA3, ura3:imm434/ura3:imm434 ssk1:hisG/ssk1:hisG-URA3-his }\end{array}$ & ire1DXssk1 $\Delta / \Delta$ & This study \\
\hline $\mathrm{JC2171}$ & $\begin{array}{l}\text { (RM1000) loxP, + pACT1-HOG1-GFP-NLS hog1:loxP-ura3-loxP, } \\
\text { hog1:loxP-HIS1-loxP, + pACT1-HOG1-GFP-NLS }\end{array}$ & Hog1-GFP-NLS & Day et al., 2017 \\
\hline JC2172 & $\begin{array}{l}\text { (RM1000) loxP, + pACT1-HOG1-GFP-CaaX hog1:loxP-ura3-loxP, } \\
\text { hog1:loxP-HIS1-loxP, + pACT1-HOG1-GFP-CaaX }\end{array}$ & Hog1-GFP-CaaX & Day et al., 2017 \\
\hline
\end{tabular}

sgRNA/F_SSK1 and sgRNA/R). In the second round, primer extension was performed to fuse PCR 1 and 2 and, finally, in the third step, PCR with nested primers SNR52/N and sgRNA/N was performed to generate the sgRNA expression cassette. An ssk1 1 :NAT1 construct for the complete deletion of SKK1 had 80-bp arms homologous to sequences upstream (89-bp from the start codon) and 9-bp immediately downstream the stop codon and the NAT1 marker for selection. ire1 DX mutant strain (Table 1) was transformed with $s s k 1 \Delta: N A T 1$ construct and PCR products and nourseothricin resistant transformants were selected and analyzed for SSK1 deletion by PCR genotyping. We checked for SSK1 deletion (using primers Comp_SSK1_del_F and Comp_SSK1_del_R) and the absence of SSK1 wild type gene (using primers Up-comp-SSK1 and Comp_SSK1_del_R). From six colonies analyzed in ire 1 background, 50\% of them had both alleles deleted and 50\% just one. Primers used in the study are listed in Supplementary Table 1.

\section{Drug Susceptibility and Viability Assays}

Candida albicans strains were grown overnight on YEPD plates at $30^{\circ} \mathrm{C}$. Cells were then diluted in $5 \mathrm{ml}$ of $0.9 \%$ saline solution to $\mathrm{OD}_{600}$ of 0.1 . Once the OD was set at 0.1 , the culture was diluted 10 times with saline and subject to 5-fold serial dilutions. Then, $5 \mu \mathrm{l}$ of culture from each of the dilutions was finally spotted on agar plates containing indicated drugs. The plates were incubated at $30^{\circ} \mathrm{C}$ and photographed after $48 \mathrm{~h}$.

For viability assay, Candida strains were grown in YEPD medium overnight. The cells were resuspended in fresh YEPD medium to an $\mathrm{OD}_{600}$ of 0.3 and grown until $\mathrm{OD}_{600}$ of 1.0 at $30^{\circ} \mathrm{C}$.
Exponentially growing cells were collected by centrifugation and resuspended in YEPD containing $20 \mu \mathrm{g} / \mathrm{ml}$ tunicamycin and kept at $30^{\circ} \mathrm{C}$. Samples were taken at different time points and spotted onto YEPD plates $\left(10^{5}\right.$ cells in $\left.10 \mu \mathrm{l}\right)$ and incubated for $24 \mathrm{~h}$ at $30^{\circ} \mathrm{C}$.

\section{Protein Extraction and Immunoblot Analysis}

For the Hog1 phosphorylation blot, overnight cultures were diluted in fresh YEPD media to an $\mathrm{OD}_{600}$ of 0.3 and grown until they reached an $\mathrm{OD}_{600}$ of 1.0 at $30^{\circ} \mathrm{C}$. Lysates were prepared in ice-cold lysis buffer $(150 \mathrm{mM} \mathrm{NaCl}, 1 \%$ Triton X$100,0.1 \%$ SDS, $1 \mathrm{mM}$ phenylmethylsulfonyl fluoride and protease inhibitor mixture) using acid-washed glass beads. The cell lysate was centrifuged at $4000 \mathrm{rpm}$ for $2 \mathrm{~min}$ at room temperature, and the supernatant was used for further analysis. Protein concentration of lysates was estimated using BCA protein assay kit (BioBasic, SK3021). Then, $50 \mu \mathrm{g}$ of the protein samples were separated by $10 \%$ sodium dodecyl sulfate-polyacrylamide (SDSPAGE) gel electrophoresis. Protein was transferred to PVDF membrane (0.45 $\mu \mathrm{m}$ pore size; Merck-millipore) at $90 \mathrm{~V}$ for 2 to $3 \mathrm{~h}$ followed by blocking with 3\% BSA (SRL) in TrisBuffered saline (TBS) with $0.1 \%$ Tween-20 for $1 \mathrm{~h}$. The blot was then probed with primary antibody 1:1000 for anti-Phospho Hog1 (phospho-p38 MAPK antibody, Thr180/Tyr182; \#9215; Cell Signaling Technology) and 1:300 for anti-Hog1 (A-8: sc16597, Santa Cruz Biotechnology) at $4^{\circ} \mathrm{C}$ overnight followed by washing thrice with TBST (TBS $+0.1 \%$ Tween-20). Secondary HRP-conjugated antibody (Goat rabbit IgG-HRP Sigma/Goat 
anti-mouse IgG-HRP; Sigma) was then added at a dilution of 1:5000 for $1 \mathrm{~h}$, followed by three washes with TBST. The blot was developed on an X-ray film using ECL reagent (BioRad Clarity ${ }^{\mathrm{TM}}$ Western ECL substrate).

\section{Fluorescence Microscopy}

Yeast strains were grown at $30^{\circ} \mathrm{C}$ in YEPD medium to an $\mathrm{OD}_{600}$ of 0.1 . In the case of treated cells, $\mathrm{NaCl}$ or tunicamycin was added to the concentration as described and $1 \mathrm{ml}$ samples were taken at different time points (30-180 $\mathrm{min}$ ) after the addition of $2 \mu \mathrm{g} / \mathrm{ml}$ of tunicamycin or $5 \mathrm{~min}$ after exposure to $1 \mathrm{M} \mathrm{NaCl}$ (positive control). Samples were centrifuged at $6000 \mathrm{rpm}$ for 2 min and washed twice with 1X PBS. Cells were fixed with 3.7\% formaldehyde (kept at $-20^{\circ} \mathrm{C}$ ) for $5 \mathrm{~min}$, centrifuged at $6000 \mathrm{rpm}$ for $1 \mathrm{~min}$, and washed twice with $1 \mathrm{X}$ PBS. DAPI $\left(4^{\prime}, 6\right.$-diamidino2-phenylindole) was added to a final concentration of $1 \mu \mathrm{g} / \mathrm{ml}$ to stain the DNA and incubated at least for $15 \mathrm{~min}$. Digital images were captured by confocal using a $100 \times$ oil objective lens.

\section{RNA Extraction and Quantitative Real-Time PCR}

Candida albicans strains were grown overnight in YEPD, subcultured from a starting $\mathrm{OD}_{600}$ of 0.3 in fresh YEPD and incubated at $30^{\circ} \mathrm{C}$ until $\mathrm{OD}_{600}$ reached 1.0. The desired drugs/compounds of interest were added to the media and culture was allowed to grow for indicated time periods. Cells were collected by centrifugation at $5000 \mathrm{rpm}$ for $5 \mathrm{~min}$ at $4^{\circ} \mathrm{C}$ from treated and untreated control samples and total RNA was isolated using the RNeasy mini kit (Qiagen). Extracted RNA was treated with DNase I (Thermo Scientific) to remove contaminating DNA and $c D N A$ was synthesized with a RevertAid $^{\mathrm{TM}} \mathrm{H}$ Minus First Strand cDNA synthesis kit (Thermo Scientific) following the manufacturer's protocol. All real-time polymerase chain reaction (PCR) reactions were performed in a volume of $25 \mu \mathrm{l}$ using Thermo Scientific Maxima SYBR Green mix in a 96-well plate. For relative quantification of gene expression, the comparative $\mathrm{C}_{T}$ method was used. Fold change (treated/untreated) is calculated by $2^{-\Delta \Delta C_{T}}$, normalized to ACT1 (endogenous control) with untreated wild type or mutant strains as calibrators. All values are mean \pm S.D and are derived from three independent RNA preparations. The data were analyzed statistically using Student's $t$-test. A $p \leq 0.05$ was considered statistically significant. The qRT-PCR primers used in this study were designed by Primer Express 3.0 and are listed in Supplementary Table 2 .

\section{Determination of HAC1 mRNA Splicing}

Candida albicans cells were treated with tunicamycin for different time points and total RNA was prepared by following the procedure described above. cDNA was prepared by using RevertAid $^{\mathrm{TM}} \mathrm{H}$ Minus First Strand cDNA synthesis kit (Thermo Scientific) and HAC1 mRNA splicing was measured by the following:

(i) Reverse Transcriptase-PCR (RT-PCR) using HAC1 genespecific primers HAC1SP (F) and HAC1SP (R) (Supplementary Table 2) and the PCR product was analyzed on $4 \%$ agarose gel. (ii) qRT-PCR was performed with TaqMan probe specific for spliced $H A C 1\left(H A C 1^{s}\right)$ or primers recognizing the unspliced HAC1 $\left(H A C 1^{u}\right)$ isoform. TaqMan Universal PCR Master Mix and primer-probe mixes were obtained from Applied Biosystems by Life Technologies. ACT1 was used as the internal control and transcript level of the gene of interest was normalized to ACT1 levels. Fold changes are means \pm S.D and are derived from three independent RNA preparations.

\section{Glycerol Production Assay}

Candida strains were grown at $30^{\circ} \mathrm{C}$ in YEPD medium to an $\mathrm{OD}_{600}$ of 1.0. Cells were collected by centrifugation and resuspended in YEPD containing $2 \mu \mathrm{g} / \mathrm{ml}$ tunicamycin or $1 \mathrm{M}$ sorbitol (positive control) for $3 \mathrm{~h}$. After $3 \mathrm{~h}, 1 \mathrm{ml}$ of the culture was removed and pelleted. Then, $1 \mathrm{ml}$ of Tris- $\mathrm{Cl}(0.1 \mathrm{M})$ was added and samples were boiled for $10 \mathrm{~min}$ at $90^{\circ} \mathrm{C}$. Following, $10 \mu \mathrm{l}$ of this sample was taken for assaying the glycerol content. Glycerol was determined colorimetrically with a commercial kit (Sigma) following the manufacturer's instructions. The values obtained are expressed as $\mu \mathrm{M}$ of glycerol per $\mathrm{mg}$ of yeast cells, dry weight. For tunicamycin-treated samples, increase in glycerol content was estimated after subtraction of the values measured in the control assays containing DMSO alone. The values shown represent means \pm S.D and are derived from the average of three independent experiments.

\section{Statistical Analysis}

All the data were plotted and analyzed using GraphPad Software. Intergroup comparisons were made using the Student's $t$-test. $P$ value of $\leq 0.05$ was considered significant.

\section{RESULTS}

\section{High-Osmolarity Glycerol Pathway Mediates Endoplasmic Reticulum Stress Resistance}

Considering the role of HOG pathway during tunicamycin (Tm)induced ER stress resistance in S. cerevisiae (Torres-Quiroz et al., 2010), we were interested in probing the role of the C. albicans HOG pathway during ER stress. To this end, we first tested the $\operatorname{hog} 1 \Delta / \Delta$ cells for growth in presence of wellknown ER stressors such as Tm, a natural inhibitor of $N$-linked glycosylation, dithiothrietol (DTT), and $\beta$-mercaptoethanol, all of which lead to misfolding of proteins in the ER, thus resulting in ER stress. YEPD supplemented with $1 \mathrm{M} \mathrm{NaCl}$ (osmostressor) was used as control because $\operatorname{hog} 1 \Delta / \Delta$ is known to exhibit increased susceptibility to this compound (San José et al., 1996). A conditional mutant strain, ire1 DX (CW906; Table 1), that exhibits diminished expression of IRE1 and is unable to grow in presence of Tm in C. albicans (Woolford et al., 2016; Sircaik et al., 2021) was also used as a control in the spot assays. As observed in Figure 1A, $\operatorname{hog} 1 \Delta / \Delta$ cells exhibited increased susceptibility to all the ER stressors, compared to the wild type and the reconstituted strain (Figure 1A). We also included $p b s 2 \Delta / \Delta$, which is the homozygous mutant for the 


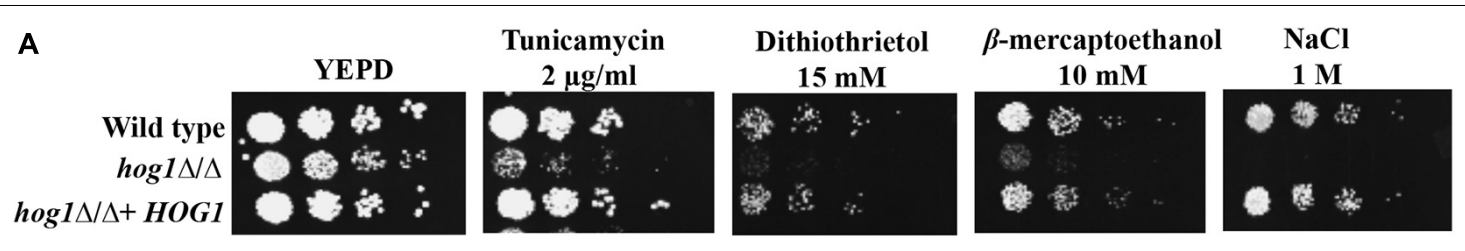

B

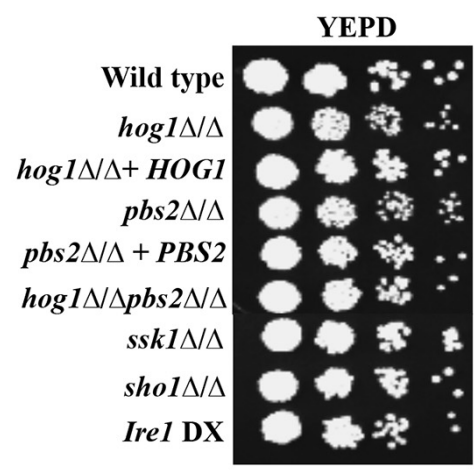

Tunicamycin $2 \mu \mathrm{g} / \mathrm{ml}$

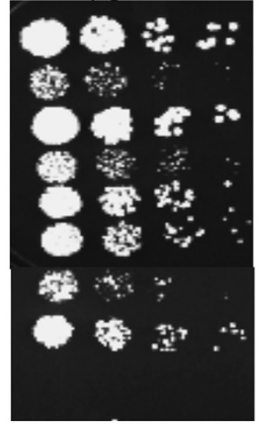

FIGURE 1 | Growth of hog1 $\Delta / \Delta$ and mutants of upstream components of HOG MAPK pathway during ER stress. (A) and (B) Serial dilutions of cell suspensions of the indicated strains were spotted onto YEPD plates supplemented with drugs at indicated concentrations and incubated at $30^{\circ} \mathrm{C}$ for $48 \mathrm{~h}$. (C) Exponentially growing cells were exposed to $20 \mu \mathrm{g} / \mathrm{ml}$ tunicamycin in liquid YEPD medium and kept at $30^{\circ} \mathrm{C}$. Samples were taken at different times and spotted onto YEPD plates $\left(10^{5}\right.$ cells in $\left.10 \mu \mathrm{l}\right)$ and incubated for $24 \mathrm{~h}$ at $30^{\circ} \mathrm{C}$.

MAPKK of the HOG pathway and hog $1 \Delta / \Delta p b s 2 \Delta / \Delta$ cells in the spot assay and observed their variable susceptibility to Tm (Figure 1B). In order to further characterize the differential behavior of $h o g 1 \Delta / \Delta, p b s 2 \Delta / \Delta$, and $\operatorname{hog} 1 \Delta / \Delta p b s 2 \Delta / \Delta$ on Tm, we performed an experiment wherein the kinetics of viability was measured for these mutants in presence of Tm. Cells from exponential phase were treated with a lethal concentration of $\mathrm{Tm}(20 \mu \mathrm{g} / \mathrm{ml})$ in liquid YEPD medium and samples were collected at different times and spotted on YEPD plates. As shown in Figure 1C, the $\operatorname{hog} 1 \Delta / \Delta$ mutant lost viability faster than not only the wild type but also the pbs $2 \Delta / \Delta$ and hog $1 \Delta / \Delta p b s 2 \Delta / \Delta$ mutants. Furthermore, the $p b s 2 \Delta / \Delta$ and the hog $1 \Delta / \Delta p b s 2 \Delta / \Delta$ mutants were slow in losing viability, compared to hog $1 \Delta / \Delta$ cells (Figure 1C). This set of data indicates that: (i) the ability of Hog1 to counter ER stress may be only partly dependent on the phosphorylating activity of the Pbs2 MAPKK as indicated by the decreased susceptibility of $p b s 2 \Delta / \Delta$ cells to Tm (Figure 1C), and (ii) decreased susceptibility of the hog $1 \Delta / \Delta p b s 2 \Delta / \Delta$ cells, compared to the parent mutant strains, could be through the activation of alternate pathways consistent with the role of a functional Hog1 in repressing cross-talk with other MAPK pathways (Eisman et al., 2006; Cheetham et al., 2011).
HOG pathway operates via two distinct branches. The first branch consists of the two-component protein, SIn 1 and the Ypd1 and Ssk1 phosphorelay system, while the second branch operates through the transmembrane adaptor protein Sho1 (Alonso-Monge et al., 2006). We therefore assessed the contribution of these branches toward the survival of C. albicans during ER stress and included the $s s k 1 \Delta / \Delta$ and sho $1 \Delta / \Delta$ mutants in Tm susceptibility assay. The ssk $1 \Delta / \Delta$ mutant displayed increased susceptibility to Tm, compared to the sho1 $\Delta / \Delta$ mutant (Figure 1B), indicating that Hog1mediated ER stress resistance may have an increased dependency on the Ssk1 response regulator of the HOG pathway in C. albicans. Taken together, this set of data points to the requirement of HOG MAPK pathway during ER stress in C. albicans.

\section{SSK1 and the Canonical Unfolded Protein Response Pathway Mediate Hog1 Activation During Endoplasmic Reticulum Stress}

To investigate the involvement of Hog1 in the ER stress response, we examined the phosphorylation status of Hog1 in 
wild type cells following exposure to $\mathrm{Tm}(2 \mu \mathrm{g} / \mathrm{ml})$. Hog1 phosphorylation was induced significantly after $2 \mathrm{~h}$ treatment with Tm (Figure 2A), pointing to the contribution of Hog1 during late phase of ER stress in C. albicans. To investigate if phosphorylated Hog1 accumulates in the nucleus, we monitored the localization of Hog1-GFP in wild type C. albicans cells. For this, the cells were treated with $\mathrm{Tm}(2 \mu \mathrm{g} / \mathrm{ml})$ up to $3 \mathrm{~h}$ and $1 \mathrm{M} \mathrm{NaCl}$ for $5 \mathrm{~min}$, and samples were collected at different time points. Hog1-GFP accumulated in the nucleus rapidly, following exposure to $1 \mathrm{M} \mathrm{NaCl}$ for $5 \mathrm{~min}$. Contrastingly, while moderate accumulation of Hog1-GFP was detected in the cytosol, it failed to accumulate in the nucleus, following Tm exposure for $1 \mathrm{~h}$ (Figure 2B). At time points later than $1 \mathrm{~h}$, Hog1-GFP was detected in the nucleus with a concomitant enrichment of GFP in the cytosol in agreement with its phosphorylation status (Figure 2B). This data suggests that: (i) ER stress-induced Hog1 phosphorylation and its subsequent translocation to the nucleus is a less pronounced and slow process, compared to the response of Hog 1 to osmotic stress and (ii) Hog 1 may be required specifically during late phase of ER stress in C. albicans.

Hog1 is activated by Pbs2 MAPKK-dependent phosphorylation resulting in its migration to the nucleus in response to osmotic stress (Alonso-Monge et al., 2003; Smith et al., 2004; Arana et al., 2005). We therefore examined Hog1 phosphorylation levels in pbs $2 \Delta / \Delta$ cells following exposure to $\operatorname{Tm}(2 \mu \mathrm{g} / \mathrm{ml} ; 3 \mathrm{~h})$. Hog1 phosphorylation was abrogated in pbs $2 \Delta / \Delta$ cells (Figure $2 \mathrm{C}$ ), indicating that Hog1 is phosphorylated in a Pbs2-dependent manner during ER stress in C. albicans.

Considering that Ire1 is the sole sensor of ER stress in fungi (Krishnan and Askew, 2014), we questioned its mode of communication with the HOG pathway during ER stress. To answer this question, Hog1 phosphorylation was checked in hac $1 \Delta / \Delta$ and ire1 DX mutant strain treated with Tm $(2 \mu \mathrm{g} / \mathrm{ml}$; $3 \mathrm{~h}$ ), two main components of the conserved UPR pathway in this fungus. Both the mutants exhibited significantly reduced levels of phosphorylated Hog1, indicating toward the essentiality of functional UPR pathway for full-blown activation of the HOG pathway during ER stress (Figure 2D).

Given that ER stress is one of the underlying causes for alterations in cell wall integrity (Krysan, 2009; Sircaik et al., 2021), it is possible that activation of Hog1 during ER stress is an indirect effect of cell wall stress. Moreover, Hog1 is also activated by osmotic stress in C. albicans (Alonso-Monge et al., 2003), questioning again the direct effect of ER stress on the activation of Hog1. To answer this question, ire1 DX mutant strain was treated with $1 \mathrm{M} \mathrm{NaCl}(10 \mathrm{~min})$ and Calcofluor white $(50 \mu \mathrm{g} / \mathrm{ml} ; 2 \mathrm{~h})$ to induce osmotic and cell wall stresses, respectively. While the activation of Hog1 remained unaffected during osmotic stress (Figure 2E), Hog1 phosphorylation in ire1 DX mutant strain was slightly enhanced in response to the cell wall stressor (Figure 2F), in accordance with previous reports that demonstrate an exacerbated cell wall stress in the absence of functional UPR (Krysan, 2009; Sircaik et al., 2021). This result was in contrast to the significantly reduced Hog1 phosphorylation in ire1 DX mutant strain during Tm-induced ER stress (Figure 2D). Thus, these results suggest that C. albicans utilizes a mechanism, discrete from that of osmotic or cell wall stress, to induce Hog 1 phosphorylation during late phase ER stress. Considering that the absence of Ire1 and Hac1 resulted in diminished but not abrogated phosphorylation of Hog1, our result points to the contribution of a functional Ire 1 in regulating the activity of the HOG pathway during ER stress.

Given that the HOG pathway is composed of Shol- and Sln1-dependent branches that are responsive to different stresses, we sought to identify its mediator branch during Tm-induced ER stress. Following exposure to Tm, sho1 $\Delta / \Delta$ cells showed wild type levels of Hog1 phosphorylation, in contrast to the significantly reduced phosphorylation of Hog1 in cells lacking SSK1 (component of Sln1 branch) (Figure 2G). This is suggestive of a prominent role of the Ssk1 response regulator in mediating Tm-induced ER stress in C. albicans.

To define the relationship between the Ire1-dependent UPR pathway and the Ssk1-mediated activation of HOG pathway during ER stress, we created a ire1DXssk1 $\Delta / \Delta$ (Table 1) double mutant strain. Hog1 phosphorylation was completely eliminated in the double mutant (Figure 2G). Taken together, these results indicate the dependency of Hogl phosphorylation on a functional Ire1-dependent UPR and the Ssk1 mediator branch during ER stress. All told, we propose that Hog1 phosphorylation during ER stress is not the result of indirect activation of osmotic stress or cell wall stress pathways and UPR and HOG pathways operate in parallel to combat ER stress in C. albicans.

\section{Hog1 Terminates Ire1-Dependent Unfolded Protein Response to Promote Adaptation to Endoplasmic Reticulum Stress}

Given the requirement of functional Ire1 for Tm-induced fullblown activation of Hog1, we were prompted to analyze the basis of the cross-talk between the HOG and canonical Ire1Hac1-dependent UPR pathways. Candida albicans depends on the prompt activation of the canonical UPR to abate early waves of ER stress (Sircaik et al., 2021). Subsequently, when the ER function is restored, adaptation to ER stress occurs, and the UPR is terminated. UPR activity is monitored by analyzing the kinetics of appearance and disappearance of the $H A C 1^{s}$ (spliced) mRNA. During late stage of ER stress, the disappearance of the $H A C 1^{s}$ mRNA reflects the timely termination of the UPR activity that is shown to correlate with recovery of ER functions and, thus, adaptation to ER stress in S. cerevisiae (Chawla et al., 2011). Therefore, to test for a relationship between HOG and Ire1-Hac1-dependent UPR pathways, we traced Ire1 activity by monitoring the processing of $\mathrm{HACl}^{u}$ (unspliced) mRNA and the transcriptional induction of the Hacl-dependent genes (KAR2, SEC61, and YSY6) in the wild type and hog1 $\Delta / \Delta$ cells (Wimalasena et al., 2008; Thomas et al., 2015; Sircaik et al., 2021). We monitored the induction of the aforesaid genes, following exposure of wild type and hog $1 \Delta / \Delta$ cells to Tm for $1 \mathrm{~h}$ and $3 \mathrm{~h}$, as phosphorylation of Hog 1 was also examined at these same time points (Figure 2A). Our analysis shows that gene expression in $\operatorname{hog} 1 \Delta / \Delta$ cells remain unchanged relative to the wild type following Tm exposure for $1 \mathrm{~h}$ (Figure 3A). However, 


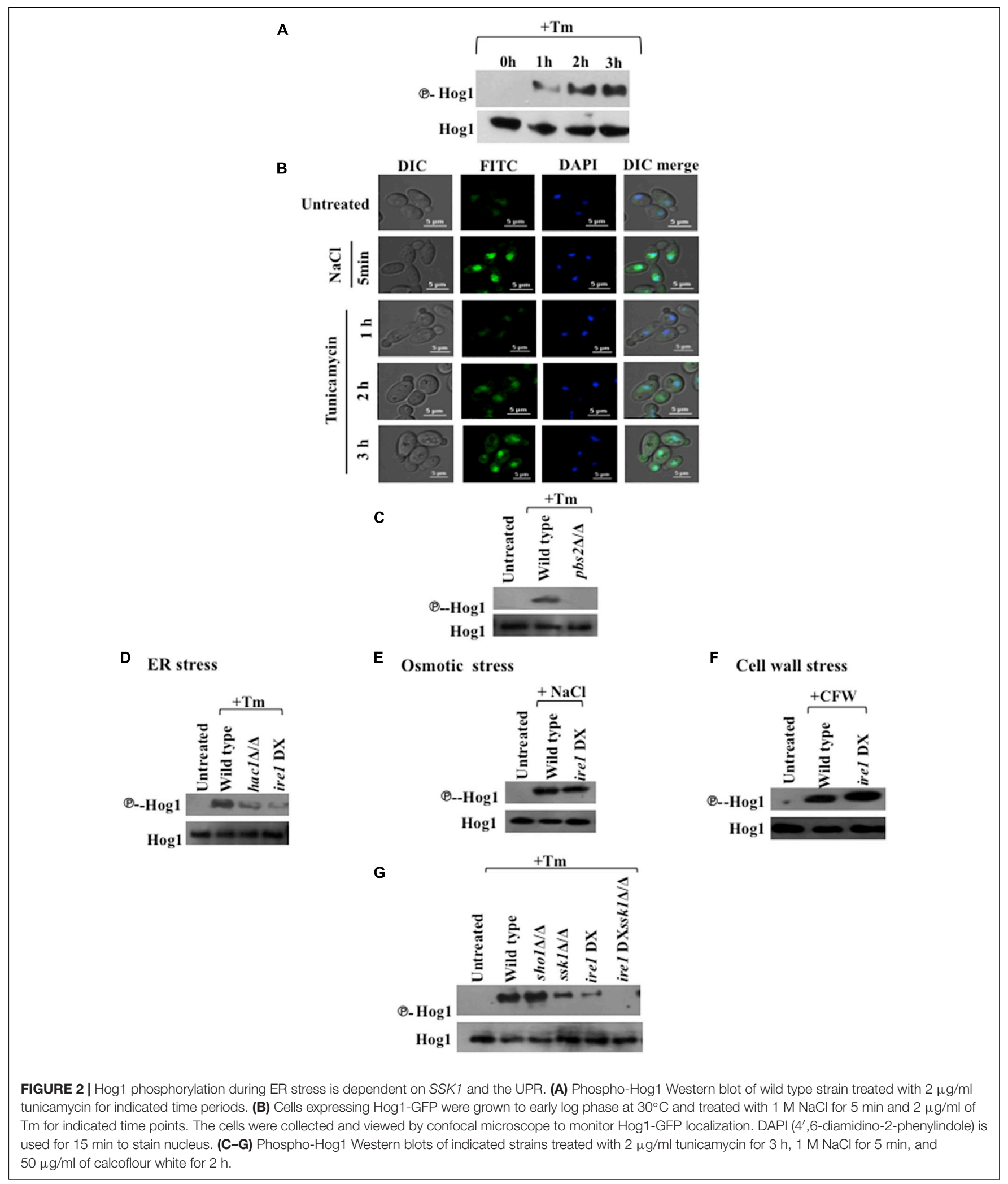

at $3 \mathrm{~h}$, the transcript levels of KAR2, SEC61, and YSY6 were 1. 7-, 1. 7-, and 1.4-fold upregulated, respectively, in the mutant compared to the wild type (Figure 3A). We hypothesized that the increased expression of the Hac1-dependent genes in $\operatorname{hog} 1 \Delta / \Delta$ cells may be due to an alteration in the kinetics of UPR activation. To this end, the kinetics of appearance of the $H A C 1^{s}$ (spliced) 

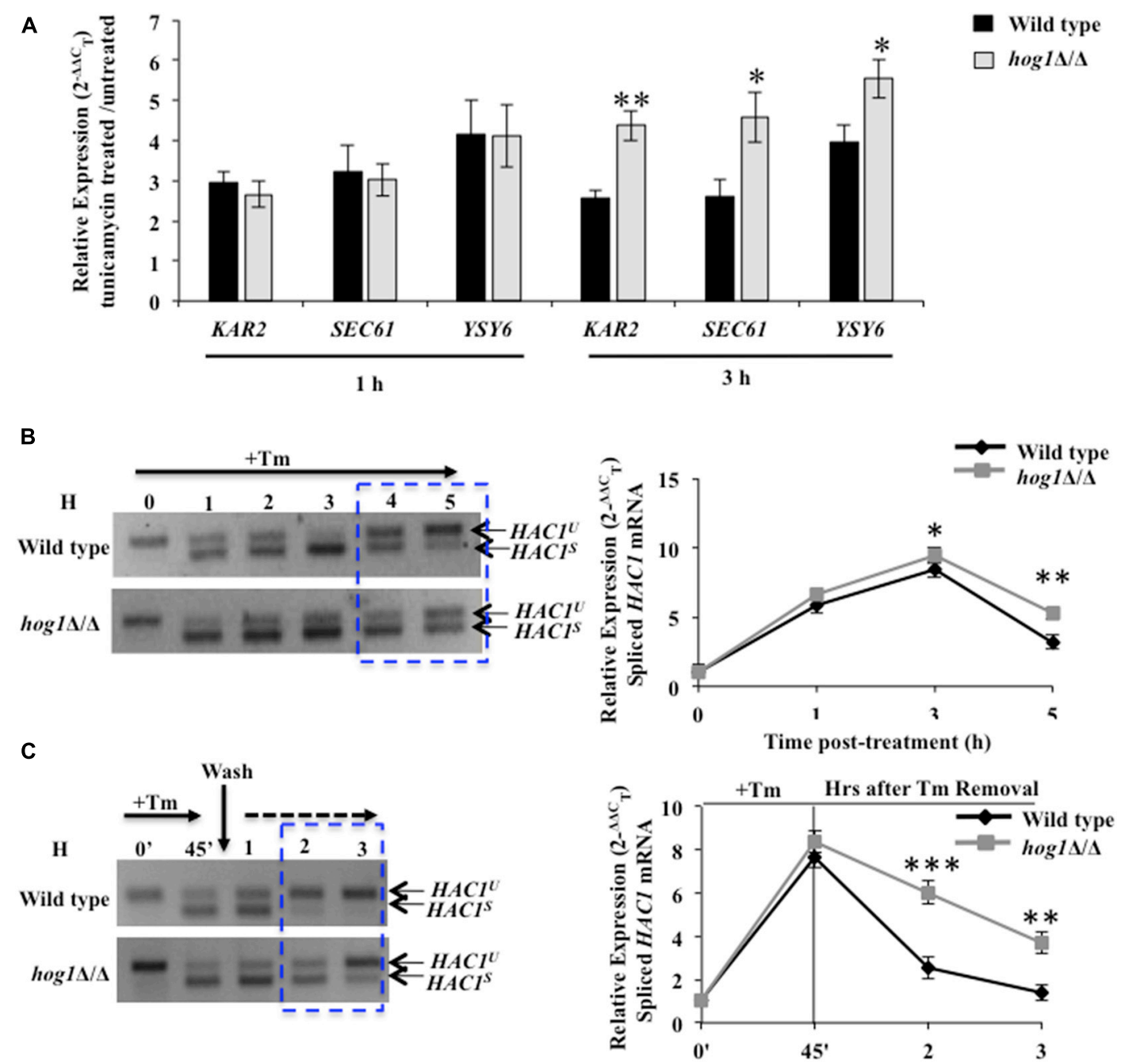

FIGURE $3 \mid$ hog $1 \Delta / \Delta$ retains high levels of spliced HAC1 mRNA during ER stress. (A) qRT-PCR of HAC1-dependent genes in wild type and hog1 $\Delta / \Delta$ cells following exposure to $2 \mu \mathrm{g} / \mathrm{ml}$ of tunicamycin for $1 \mathrm{~h}$ and $3 \mathrm{~h}$. (B) and (C) $H A C 1 \mathrm{mRNA}$ analysis in wild type and hog $1 \Delta / \Delta$ exposed to $2 \mu \mathrm{g} / \mathrm{ml}$ of tunicamycin for indicated time points. Total RNA was isolated from the indicated strains, cDNA was prepared and subject to PCR using HAC1 specific splicing primers; $4 \%$ agarose gel was run to check the PCR products. Graphs depict QRT-PCR with TaqMan probe specific for spliced HAC1 (HAC1's) or unspliced HAC1 isoform (HAC14) in wild type and hog $1 \Delta / \Delta$ following tunicamycin exposure. Values are the means of at least three independent experiments. The data were analyzed statistically using Student's $t$-test: * $p \leq 0.05 ;{ }^{*} p \leq 0.01 ; * * * p \leq 0.001$.

mRNA in $\operatorname{Tm}(2 \mu \mathrm{g} / \mathrm{ml})$ treated wild type and hog $\Delta / \Delta$ cells was examined by (a) performing RT-PCR using primers across the HAC1 mRNA intron followed by analyzing the PCR products on agarose gel and (b) using Taqman probes for higher efficiency during target amplification. The $H A C 1^{s}$ mRNA levels appeared in both the wild type and the mutant within $1 \mathrm{~h}$ of Tm treatment as inferred from examining the PCR products on agarose gel (Figure 3B). This observation resonated with the 6-fold increase in the $H A C 1^{s}$ mRNA levels within $1 \mathrm{~h}$ of Tm treatment, measured by the Taqman probe analysis in wild type as well as the mutant (Figure 3B). Consistently, transcriptional induction of Hac1dependent genes occurred to similar levels in both the wild type and the mutant (Figure $\mathbf{3 A}$ ) at $1 \mathrm{~h}$. After $3 \mathrm{~h}$, there was a decrease in the level of $H A C 1^{s}$ mRNA with a concurrent increase in the $H A C 1^{u}$ mRNA levels in the wild type (Figure 3B).
In contrast, $\operatorname{hog} 1 \Delta / \Delta$ cells exhibited increased $H A C 1^{s}$ mRNA and decreased $H A C 1^{u}$ mRNA at the $4 \mathrm{~h}$ and $5 \mathrm{~h}$ time points (Figure 3B). In agreement with this observation, Taqman probe analysis demonstrated the presence of 2-fold elevated levels of $H A C 1^{s}$ mRNA in the hog $1 \Delta / \Delta$ cells, compared to the wild type at $5 \mathrm{~h}$ (Figure $3 \mathrm{~B}$ ). This result suggests that while the decline of the $H A C 1^{s}$ mRNA levels in the wild type was rapid, hog $1 \Delta / \Delta$ cells retained the $H A C 1^{s}$ mRNA levels for a longer duration. This observation correlated well with the increased transcriptional induction of the Hac1-dependent genes following Tm exposure for $3 \mathrm{~h}$, compared to the wild type (Figure 3A). The slow kinetics of disappearance of $H A C 1^{s}$ mRNA in the mutant may reflect delayed termination of the UPR and adaptation to ER stress.

To further test the correlation between the disappearance of $H A C 1^{s}$ mRNA and adaptation to ER stress, we performed UPR 
recovery experiments. We presumed that if disappearance of $H A C 1^{s}$ mRNA occurs due to attenuation of UPR, then removing the stressor (Tm) followed by growth of the wild type in medium lacking Tm should enhance the disappearance of $H A C 1^{s}$ mRNA. However, if the absence of Hogl affects the timely attenuation of UPR, then the kinetics of disappearance of $H A C 1^{s}$ mRNA should be slower in the mutant. To this end, strains of interest were treated with $\mathrm{Tm}$ for $45 \mathrm{~min}$ followed by their transfer to fresh growth medium lacking Tm. At $3 \mathrm{~h}$ after Tm washout, spliced HAC1 mRNA levels declined by 8 -fold in the wild type, compared to its levels at the start of the washout (45 min, following Tm exposure) (Figure 3C). The rapid decline was well reflected in Lane 5 of Figure $3 \mathrm{C}$ as spliced HAC1 mRNA levels were mostly undetectable at $3 \mathrm{~h}$, thus forging a link between the rapid disappearance of spliced $H A C 1$ mRNA with the timely attenuation of the UPR. In contrast, $\operatorname{hog} 1 \Delta / \Delta$ cells exhibited only a 2-fold decline in the spliced HAC1 mRNA levels at $3 \mathrm{~h}$ after Tm washout, compared to the wild type (Figure 3C). This observation was in agreement with the RT-PCR result, providing further support to our hypothesis that the slow disappearance of $H A C 1^{s}$ mRNA in the absence of Hogl reflects late attenuation and thus, recovery from ER stress in C. albicans. Altogether, we conclude that during ER stress: (i) the activity of the HOG pathway modulates the UPR pathway such that the absence of functional Hog1 causes sustained activation of the latter; and (ii) the role of Hog1 during ER stress could be tied to its ability to promptly terminate Ire1-dependent UPR for restating ER homeostasis in C. albicans.

\section{Hog1 Influences Adaptation to Endoplasmic Reticulum Stress by Regulating the Basal Levels of Glycerol in Candida albicans}

Prior studies in S. cerevisiae show that the protective effect of the HOG pathway during osmotic and ER stresses can be partially attributed to its cytosol-dependent role in regulating glycerol production. Glycerol is a natural osmolyte shown to protect cellular proteins from aggregation, during hostile conditions (Albertyn et al., 1994; Hohmann, 2002; Kumar, 2009; Torres-Quiroz et al., 2010; Petelenz-Kurdziel et al., 2013). Endoplasmic reticulum stress-induced accumulation of misfolded proteins activate GPD1 (glycerol-3-phosphate dehydrogenase) and GPP1/RHR2 (glycerol-3-phosphatase) genes required to produce glycerol (Figure 4A) in a Hog1-dependent manner in S. cerevisiae (Albertyn et al., 1994; Hohmann, 2002; Enjalbert et al., 2006). In C. albicans, while this MAPK induces GPD2 (isozyme of GPD1) and RHR2-mediated glycerol production during osmotic stress, their role during Tm-induced ER stress remains uninvestigated (Day et al., 2017). Considering this background, we hypothesized that aside from assisting the termination of the Ire1-dependent UPR pathway, Hog1 may also be influencing adaptation to ER stress through its ability to induce glycerol production in C. albicans. Functional Hog1 is required for regulating basal levels of both GPD2 and RHR2 as evidenced by their reduced transcript levels in untreated $h \circ g 1 \Delta / \Delta$ cells, compared to the wild type. Following Tm exposure, while the wild type exhibited 2-fold increase in the transcript levels of GPD2 and RHR2, the transcript level of these genes was significantly downregulated in hog $1 \Delta / \Delta$ cells (Figure 4B). This data points to the essentiality of functional Hog1 in regulating the transcription of GPD2 and RHR2 in basal as well as Tm-induced ER stress conditions in C. albicans.

To correlate the reduced transcript levels of the aforesaid genes with glycerol production, we measured glycerol levels in wild type, $\operatorname{hog} 1 \Delta / \Delta$, and hac1 $\Delta / \Delta$ cells exposed to Tm and sorbitol. Considering that glycerol production in $S$. cerevisiae occurs in response to osmostress (Torres-Quiroz et al., 2010), we included wild type cells exposed to $1 \mathrm{M}$ sorbitol as a control for the experiment. In the absence of $\mathrm{Tm}$, the intracellular glycerol content was reduced by 3 -fold in $h \circ g 1 \Delta / \Delta$ cells, compared to the wild type. Despite the downregulated expression of GPD2 and RHR2, when $h o g 1 \Delta / \Delta$ was exposed to Tm or sorbitol, the intracellular glycerol content increased by 2- and 7 -fold, respectively, compared to the untreated mutant strain (Figure 4C). These fold increases in the glycerol content were similar to that observed with the wild type exposed to Tm or sorbitol. This data points to the role of Hog1 in regulating the basal levels of glycerol, which coupled with the reduced transcript levels of GPD2 and RHR2 contributes to lower glycerol levels in the mutant in absence of the ER stressor. Moreover, it is plausible that ER stress-induced glycerol production may only be partially dependent on a functional Hog1 and the possibility of glycerol production in Hog1-independent manner cannot be ruled out in C. albicans (Figure 4C). Interestingly, hac1 $\Delta / \Delta$ cells exhibited glycerol content similar to the wild type, indicating absence of functional interaction between the HOG and the UPR pathways for glycerol production. Given the increased susceptibility of $h \circ g 1 \Delta / \Delta$ cells to Tm (Figure 1 ), it is likely that $\operatorname{Hog} 1$, by regulating basal levels of glycerol, may be involved in conferring protection to $C$. albicans during ER stress.

\section{Cytosolic as Well as Nuclear Activity of Hog1 Is Required for Mediating Endoplasmic Reticulum Stress Resistance}

Preventing nuclear accumulation of C. albicans Hog1 does not impair the activation of Hog1-dependent osmo-protective genes (Day et al., 2017). Thus in this fungal pathogen, nuclear accumulation of Hogl is not essential for the transcriptional regulation of osmo-responsive genes. To examine the contribution of the cytosolic and nuclear activity toward Hog1mediated ER stress resistance, we used strains that consisted of Hog1 that was either tethered to the plasma membrane (Hog1-GFP-CaaX) or constitutively nuclear (Hog1-GFP-NLS) (Day et al., 2017). While both the strains were partially able to resist ER stress, the Hog1-GFP-NLS cells conferred increased stress resistance, compared to the Hog1-GFP-CaaX carrying cells (Figures 5A,B). The inability of the Hog1-GFP-NLS cells to exhibit wild type levels of resistance to Tm pointed to the partial dependency of Hogl on its cytosolic activity for mounting an effective ER stress response. 
A

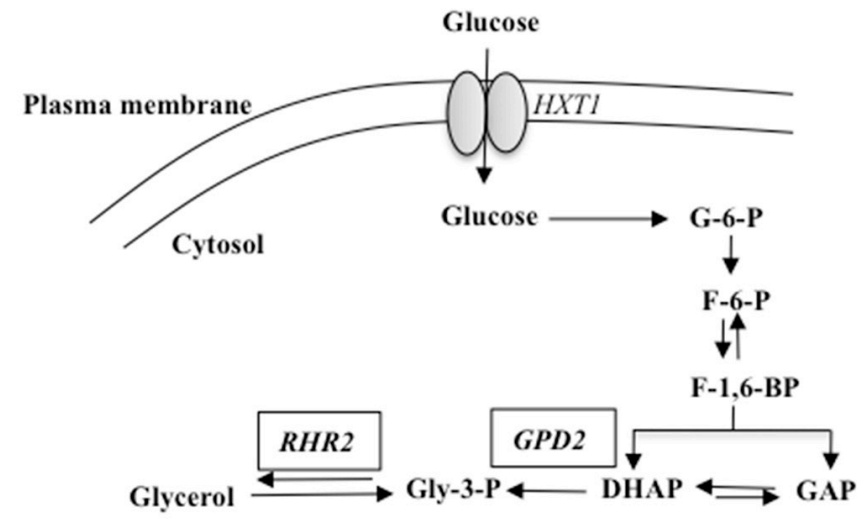

B

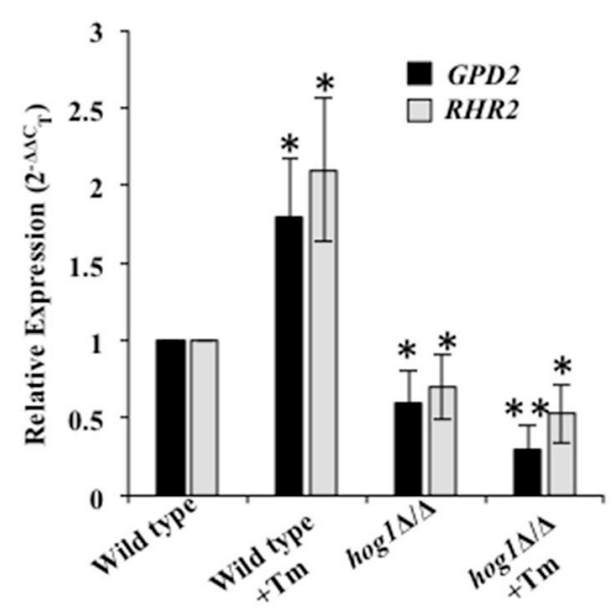

C

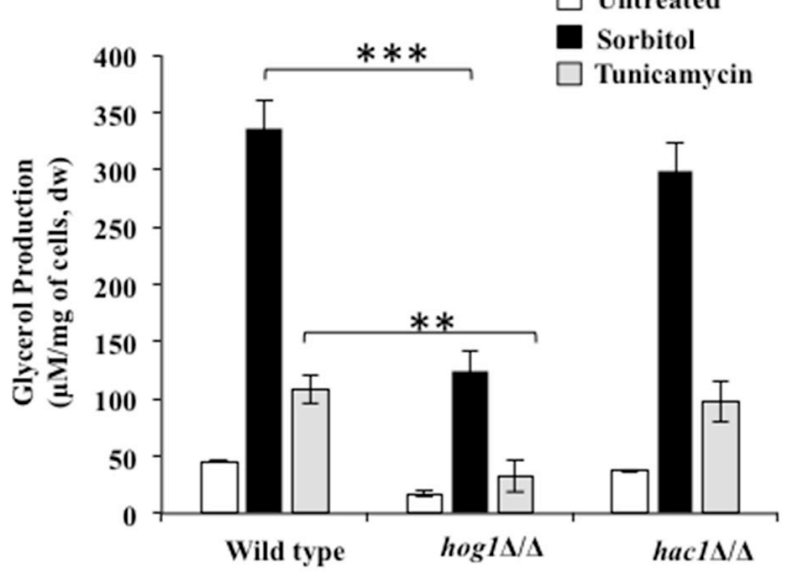

FIGURE 4 | Hog1-dependent glycerol production during ER stress. (A) Glycerol synthesis pathway in C. albicans. HXT: hexose transferase; G-6-P: Glucose-6-phosphate; F-6-P: Fructose-6-phospate; F-1, 6-BP: Fructose-1,6-biphosphate; DHAP: Dihydroxyacetone phosphate; GAP: Glyceraldehyde-3-phosphate; Gly-3-P: Glycerol-3-phosphate; RHR2: glycerol-3-phosphatase; GPD2: glycerol-3-phosphate dehydrogenase. (B) qRT-PCR to measure transcript levels of GPD2 and RHR2 following treatment $2 \mu \mathrm{g} / \mathrm{ml}$ of tunicamycin for $3 \mathrm{~h}$. (C) Wild type and indicated mutant strains were grown in YEPD at $30^{\circ} \mathrm{C}$ and then transferred to fresh medium containing $1 \mathrm{M}$ sorbitol or $2 \mu \mathrm{g} / \mathrm{ml}$ of tunicamycin. After $3 \mathrm{~h}$, aliquots ( $1 \mathrm{ml}$ ) of the cultures were withdrawn and processed for glycerol content. Values represent the difference in total glycerol before and after the indicated treatment and are the means of at least three independent experiments. dw, dry weight. The data was analyzed statistically using Student's $t$-test: * $p \leq 0.05$; ** $p \leq 0.01$; *** $p \leq 0.001$.

Because cells in which Hog1 is plasma membrane tethered retained their ability to partially resist ER stress, we speculated that the protective effect of Hog1-GFP-CaaX could be through: (i) influencing the activation of Hog1, (ii) the induction of the ER stress-specific transcriptional program, or (iii) its influence on promoting recovery from ER stress. As phosphorylation of Hog1 is a prerequisite for mediating its activity, we first sought to examine the impact of Hog1 cellular localization on its phosphorylation status following Tm exposure for $3 \mathrm{~h}$ and $5 \mathrm{~h}$. Phosphorylation of the Hog1-GFP-CaaX fusion lasted $2 \mathrm{~h}$ longer than the wild type and the Hog1-GFP-NLS fusion (Figure 5C). Thus, activation of Hog1 in response to Tm-induced ER stress is not dependent on its cellular localization. This suggests that the partial ER stress resistance exhibited by C. albicans cells expressing Hog1-GFP-CaaX could be due to increased Hog1 phosphorylation.
Second, presuming that nuclear accumulation is not essential for inducing ER stress-responsive gene expression in C. albicans, we measured the transcript levels of GPD2 and RHR2 given their Hog1-dependent expression following Tm exposure (Figure 4B). Interestingly, while the expression of GPD2 and RHR2 was induced $\geq 1.5$-fold in the wild type, their expression was abolished in the cells expressing GFP-CaaX fusion protein treated with Tm for $3 \mathrm{~h}$, correlating well with the expression profile in hog $1 \Delta / \Delta$ cells (Figure 5D). This observation shows that partial resistance of Hog1-GFP-CaaX to ER stress is not fully dependent on its nuclear translocation and the subsequent triggering of the transcriptional program. Striking was a similar abrogation of GPD2 and RHR2 in cells expressing the GFP-NLS fusion protein, suggestive of the dependency of the nuclear localized Hog 1 on the expression of Hog1-dependent cytosolic proteins for transcriptional regulation. 


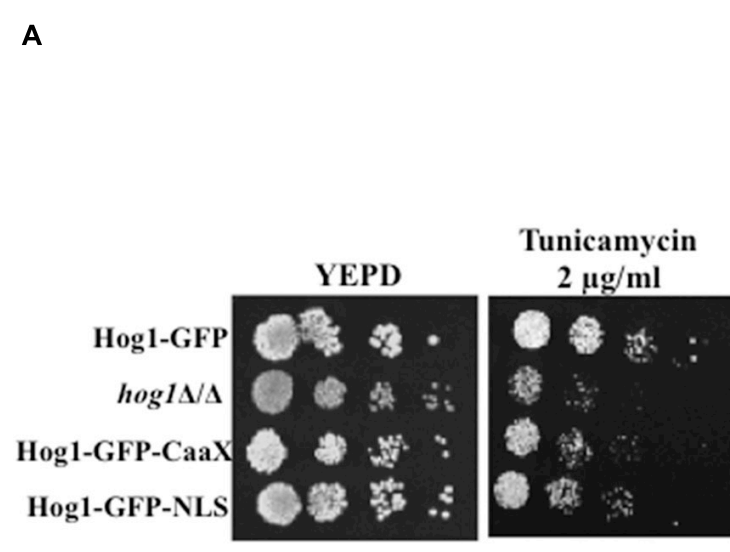

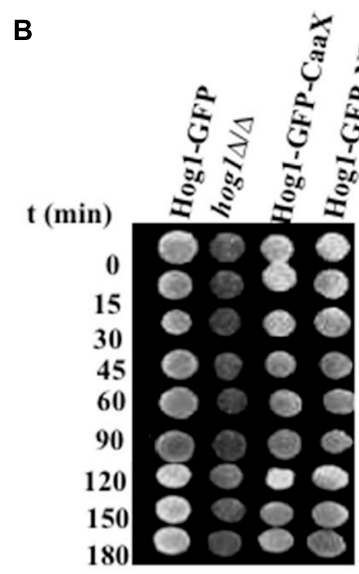

YEPD
C

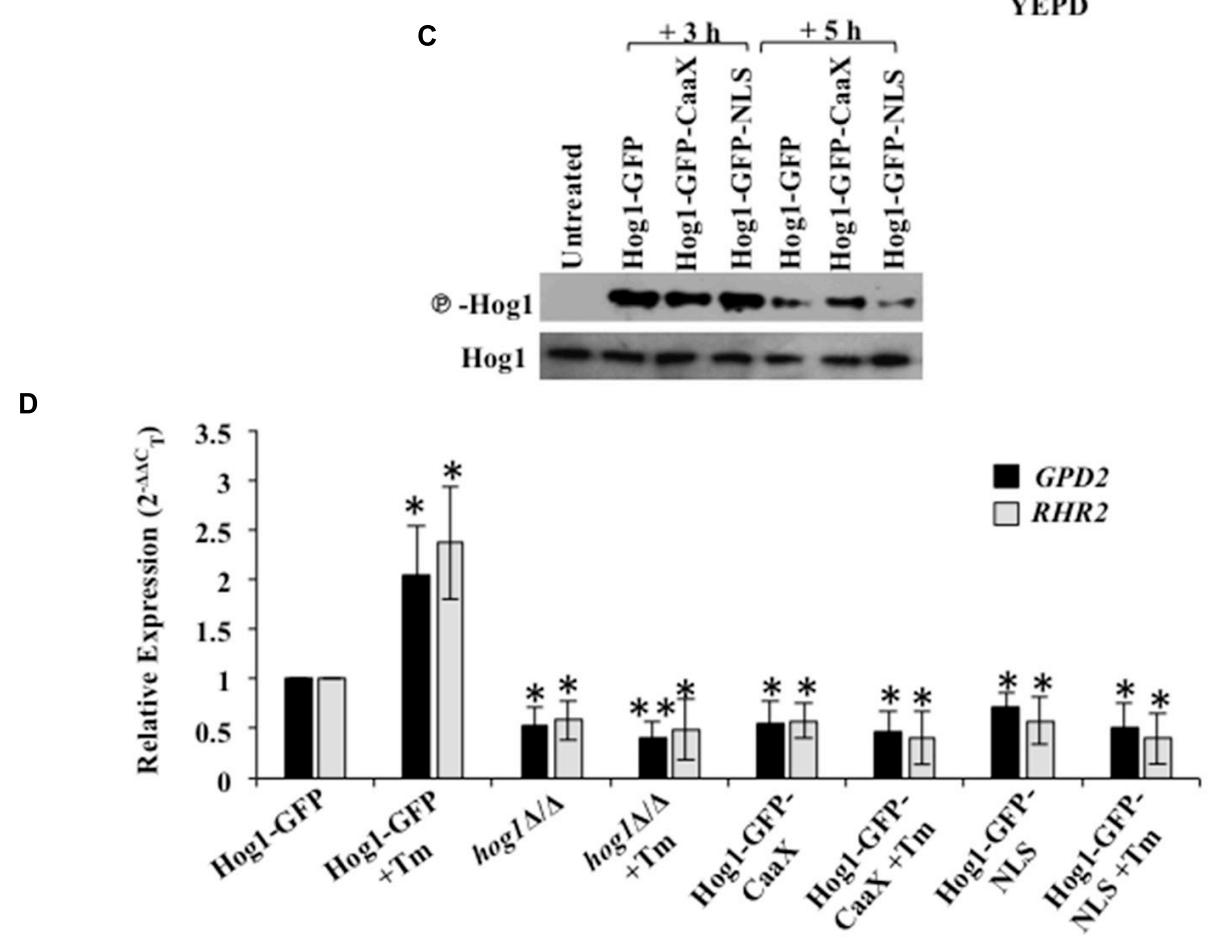

FIGURE 5 | Hog1 depends on its cytosolic and nuclear activity for ER stress resistance. (A) Serial dilutions of cell suspensions of the indicated strains were spotted onto YEPD plates supplemented with $2 \mu \mathrm{g} / \mathrm{ml}$ of tunicamycin, and incubated at $30^{\circ} \mathrm{C}$ for $48 \mathrm{~h}$. (B) Exponentially growing cells of indicated strains were exposed to $20 \mu \mathrm{g} / \mathrm{ml}$ tunicamycin in liquid YEPD at $30^{\circ} \mathrm{C}$. Samples were taken at indicated time points and spotted onto YEPD plates (10 5 cells in $\left.10 \mu \mathrm{l}\right)$ and incubated for $24 \mathrm{~h}$ at $30^{\circ} \mathrm{C}$. (C) Phospho-Hog1 Western blot of indicated strains treated with $2 \mu \mathrm{g} / \mathrm{ml}$ tunicamycin for $3 \mathrm{~h}$ and $5 \mathrm{~h}$. (D) qRT-PCR of $H O G 1$-dependent target genes (GPD2 and RHR2) upon treatment with $2 \mu \mathrm{g} / \mathrm{ml}$ of tunicamycin for $3 \mathrm{~h}$. The data was analyzed statistically using Student's $t$-test: ${ }^{*} p \leq 0.05 ;{ }^{* *} p \leq 0.01$.

Third, considering the absence of transcriptional response in C. albicans cells carrying Hog1-GFP-CaaX, we asked what other cellular process does Hog1 impact to resolve ER stress. We hypothesized that Hog1-GFP-CaaX fusion protein may be mediating ER stress resistance by promoting the timely attenuation of Ire1-dependent UPR. To address this, processing of $H A C 1^{u}$ mRNA and the subsequent activation of the UPR genes was monitored. While cells lacking HOG1 exhibited a $30 \%$ decline in $H A C 1^{s}$ mRNA levels (Figure 6A; closed squares), the wild type, Hog1-GFP-CaaX, and Hog1-GFP-NLS cells show $60 \%$ decline in $H A C 1^{s}$ mRNA levels, pointing to the slow processing of $\mathrm{HACl}^{u}$ mRNA in hog1 $\Delta / \Delta$ cells (Figure 6A). Consequentially, both the fusion proteins displayed wild type levels of inductions of the Hacl-dependent genes in Tm-treated cells for $3 \mathrm{~h}$ (Figure 6B). This indicated that C. albicans' ability to attenuate the UPR activity and promote recovery from ER stress is not impacted by its cellular localization, an attribute that may underly the partial ER stress resistance exhibited by both GFP-CaaX and -NLS cells.

Taken together, we surmise that during ER stress (i) the ability of Hog1 to activate ER stress-responsive genes may be dependent on the induction of a significant group of additional 
A
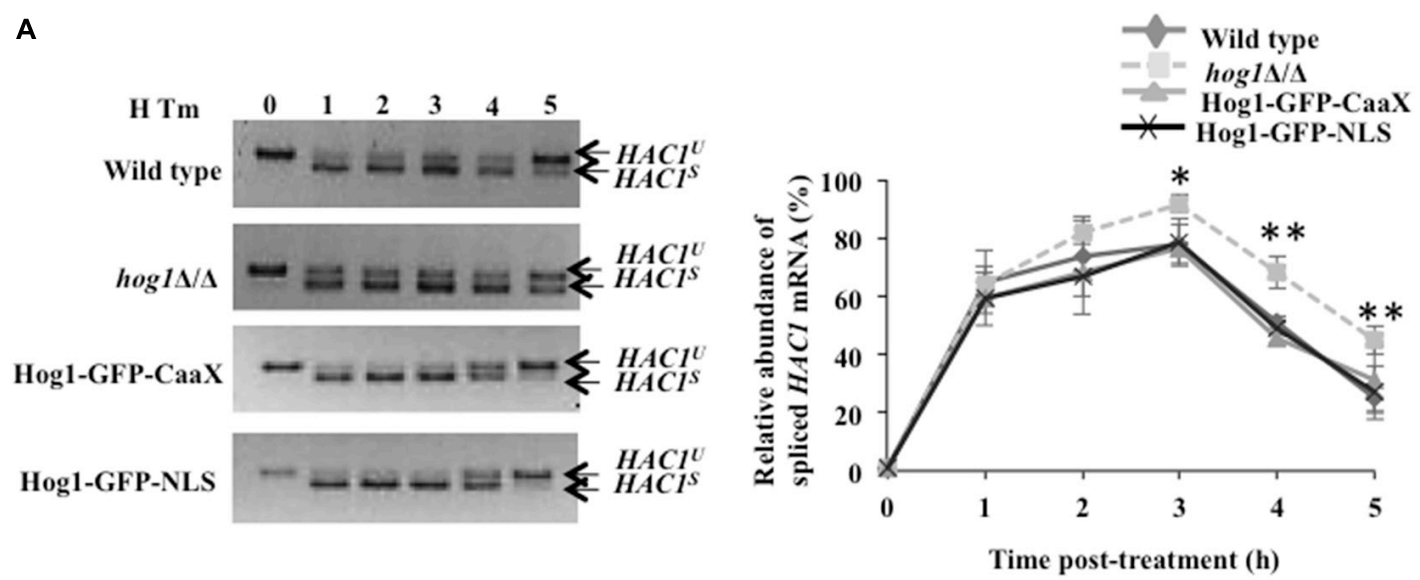

B

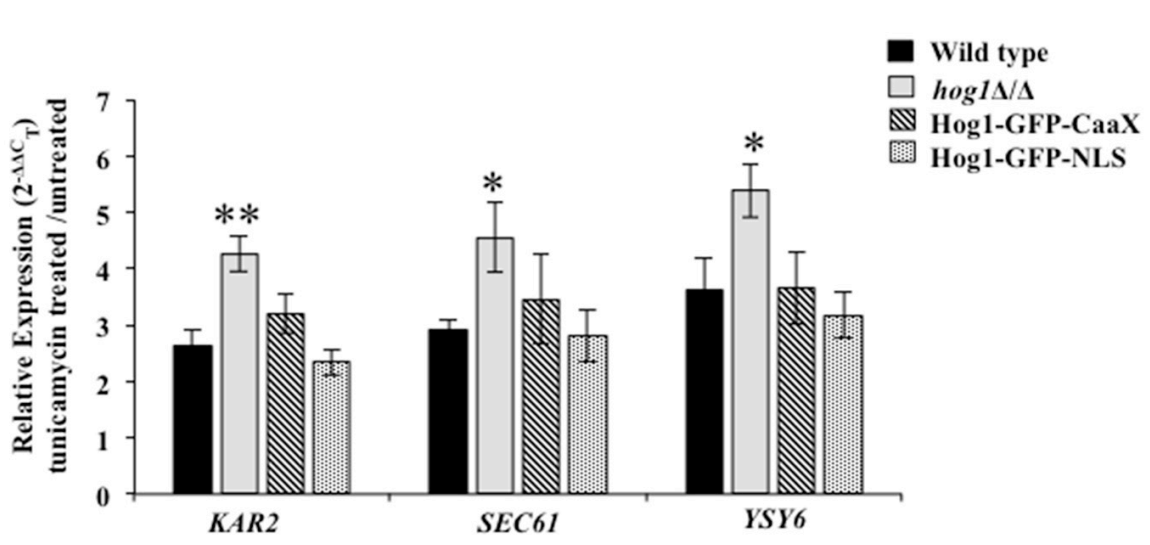

FIGURE 6 | Hog1 is dependent on both cytosolic and nuclear activity for promoting recovery from ER stress. (A) HAC1 mRNA analysis in indicated strains treated with $2 \mu \mathrm{g} / \mathrm{ml}$ of tunicamycin for the indicated time. Total RNA was isolated from the indicated strains, cDNA was prepared and 4\% agarose gel was run to check for PCR products. Graph depicts relative abundance of spliced HAC1 mRNA (\%). Values of relative abundance of spliced HAC1 mRNA were calculated by using the formula: (band intensity of HAC1 $\left.{ }^{s} \mathrm{mRNA}\right) /\left[\left(\right.\right.$ band intensity of $H A C 1^{s} \mathrm{mRNA}$ ) + (band intensity of HAC1u mRNA)]. (B) qRT-PCR of HAC1-dependent UPR target genes in indicated cells upon treatment with $2 \mu \mathrm{g} / \mathrm{ml}$ of tunicamycin for $3 \mathrm{~h}$. The data were analyzed statistically using Student's $t$-test: ${ }^{*} p \leq 0.05$; ${ }^{* *} p \leq 0.01$.

factors that require the cytosolic activity of this transcription factor, (ii) nuclear accumulation of Hog1 is not a pre-requisite for terminating the Ire1-dependent UPR activity and promoting recovery from ER stress, and (iii) cytosolic as well as nuclear activity of Hog1 are essential for mediating its protective effect during ER stress in C. albicans.

\section{DISCUSSION}

Endoplasmic reticulum (ER) stress response pathways and their regulation have a profound effect on the pathogenicity of C. albicans. The fungus relies on the activation of the Ire1dependent UPR pathway for countering ER stress, an attribute that facilitates the pathogenicity of this fungus (Sircaik et al., 2021). Subsequent sequence of events leading to the attenuation of the UPR and restoration of ER homeostasis remain an undetermined facet of the ER stress response in C. albicans. The involvement of the HOG MAPK pathway in countering multiple stresses is well established in C. albicans (Román et al., 2020).
Herein, we elucidate the position of the HOG MAPK pathway within the little known framework of UPR in C. albicans. On the basis of the increased susceptibility of cells lacking components of the HOG MAPK pathway to Tm, we inferred the involvement of this pathway in protection against ER stress in C. albicans (Figure 1). Furthermore, we propose a role of this MAPK during the late phase of ER stress in the fungus as phosphorylation of Hog1 and its nuclear enrichment was elevated following Tm exposure for extended length of time (up to $3 \mathrm{~h}$ ) (Figures 2A,B).

The results presented here uncover a functional relationship between HOG MAPK and the Ire1-dependent UPR pathways to show that parallel working of these signaling pathways is essential for adaptation and thus recovery of C. albicans from ER stress (Figure 7). During the initial phase of ER stress, after protein homeostasis is restored via the Ire1-Hac1dependent pathway, the UPR has to subside. The ribonuclease (RNase) activity-dependent processing of HAC1 mRNA is initiated and terminated by autophosphorylation followed by dephosphorylation of Ire1, respectively. Consequentially, at the later stage of ER stress, the dephosphorylation event suppresses 


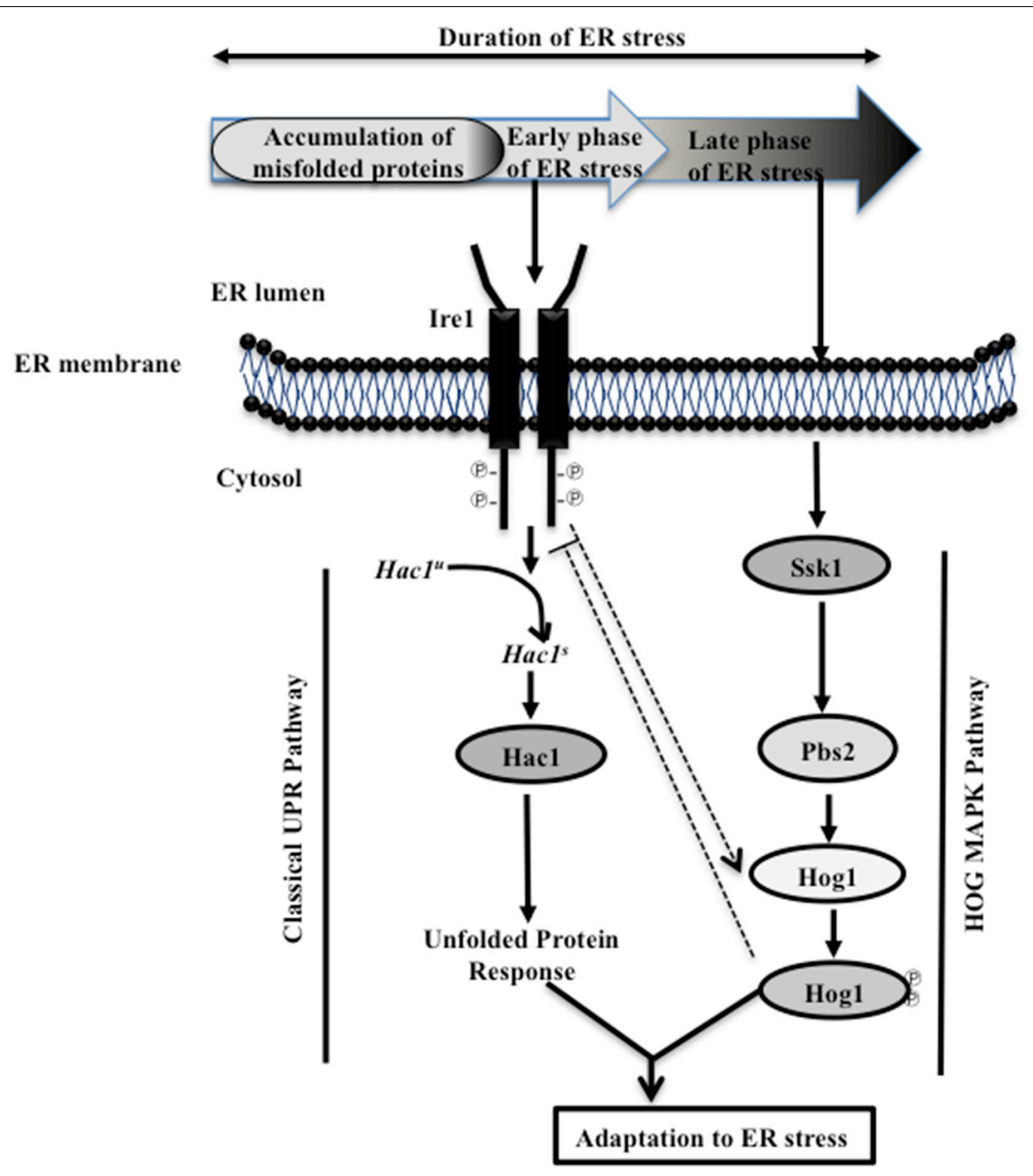

FIGURE 7 | Model depicting cellular responses during ER stress. Early (initial) exposure to ER stress initiates the autophosphorylation of Ire1, leading to the activation of its ribonuclease (RNase) activity and the subsequent splicing of the intron from HAC1 ${ }^{u}$ (unspliced) mRNA. Spliced (HAC1 ${ }^{s}$ ) mRNA codes for the Hac1 protein, which activates the UPR target genes. During ER stress, Ire1 promotes the activation of HOG MAP kinase pathway (indicated by dotted arrow). Subsequently, adaptation to ER stress is allowed, when Ssk1-mediated activation of Hog1 MAP kinase attenuates the activity of Ire1 (indicated by dotted blunt line). The attenuation of Ire1 activity could be due to the influence of Hog1, directly or indirectly, on the dephosphorylation of Ire1, which in turn regulates the RNase activity-mediated processing of HAC1 ${ }^{u}$ mRNA. As the UPR is terminated, the amplitude of the signal through the HOG pathway decreases and the stress response is fully terminated. Consequentially, the concerted action of the UPR and HOG MAPK pathways facilitate adaptation to ER stress, and guides the recovery of $C$. albicans from ER stress.

the RNase activity, thus reducing the level of spliced HAC1 mRNA and terminating the UPR in S. cerevisiae. In agreement, cells carrying mutations in the kinase domain of Ire1 exhibited elevated level of spliced HAC1 mRNA and increased susceptibility to ER stressor in the yeast (Chawla et al., 2011; Chang et al., 2018). Considering this background, we attributed the inability of $C$. albicans $h \circ g 1 \Delta / \Delta$ cells to sustain growth in the presence of Tm to its role in supporting UPR attenuation (Figure 1). This explanation is also in line with the activation of the HOG pathway solely during the late stage of ER stress (Figure 2A). Decreased levels of the spliced HAC1 mRNA after $5 \mathrm{~h}$ of Tm treatment indicated recovery from ER stress and, thus, termination of the UPR in the wild type (Figure 3B). Contrastingly, the continuous expression of spliced HAC1 mRNA $\left(H A C 1^{s}\right)$ even after the removal of the ER stressor (Figure 3C) suggested delayed termination of the UPR in $h \circ g 1 \Delta / \Delta$ cells. The influence of Hog1 in attenuating the Ire1-dependent UPR can be explained by a possible direct or indirect involvement of the MAP kinase in promoting the dephosphorylation and, thus, inactivation of Ire1, when challenged with ER stress. As a consequence, the processing of HAC1 mRNA will cease resulting in the downregulation of Hac1-dependent genes, thus enabling adaptation to ER stress. As the attenuation of the Ire1dependent UPR occurs, the amplitude of the signal through the HOG pathway may also decrease and the stress response is fully terminated (Figure 7). In agreement, compromising the expression of Ire1 (ire1 DX mutant strain) reduced the levels of phosphorylated Hog1 following Tm exposure for extended times (Figures 2B,G). One plausible explanation for this observation could be that Ire1 downregulates the expression of Hog1-specific 
phosphatases, thus allowing C. albicans to maintain normal levels of phosphorylated Hog1. Alternatively, Ire1-dependent transcriptional programming may also involve upregulating genes that facilitate Hog1 phosphorylation, aspects of ER stress that can be explored in future. We propose that by virtue of the involvement of HOG pathway in the timely attenuation of the UPR activity, the unnecessary activation of the Ire1-dependent UPR pathway is impeded, once ER homeostasis is restated, in this pathogenic fungus. Regulating the activity of Ire1-dependent UPR as a mode to modulate the ER stress response also occurs through Snf1 in S. cerevisiae. Snf1 not only downregulates the expression of Ssk1 to influence the activity of the HOG pathway but also inactivates the UPR in S. cerevisiae (Mizuno et al., 2015). This points to the existence of multiple modes of inactivating the UPR, albeit the unexplored role of Snf1 during ER stress in C. albicans.

The diversity in the upstream components that activate Hog1, the magnitude or duration of Hogl phosphorylation, and a difference in the localization pattern of this MAPK in response to various stresses influences the specificity of the downstream events (Román et al., 2020). C. albicans deploys Sln1 twocomponent protein for the activation of Hog1 during osmotic and oxidative stresses (Chauhan et al., 2003; Alonso-Monge et al., 2006; Cheetham et al., 2007). We show that during ER stress, both functional Ssk1 and Ire1 are essential for Hog1 phosphorylation and thus its activation (Figure 2G). Considering that ER and oxidative stress co-exist in yeast (Guerro-Moreno et al., 2019), it is possible that oxidative stress was the basis of Hog1 activation during prolonged ER stress. This possibility was partly ruled out as the absence of Ssk1 is shown to abolish oxidative stressinduced phosphorylation of Hog1 in C. albicans (Chauhan et al., 2003). Alternately, it is plausible that activation of anti-oxidative stress responses that are Ssk1-independent facilitate protection from oxidative stress during ER stress and that it is through this combined action of anti-ER and -oxidative stress responses that facilitate C. albicans adaptation to ER stress. If ER and oxidative stress co-exist and whether the latter modulates ER stress response in Ssk1-dependent or -independent manner are features of the UPR that remain unexplored in C. albicans. Despite the similarity in the upstream components, ER stress induced both nuclear and cytoplasmic localization of Hog1 (Figure 2B), unlike its exclusive localization in the nucleus during osmoand oxidative stresses, respectively (Smith et al., 2004; Arana et al., 2005). This was suggestive of a role for Hog1 in regulating responses to ER stress by functioning from both cytoplasm and nucleus. This observation is evidenced as follows: (i) both the cytosolic and nuclear localized Hog1 were partially able to resist growth in presence of Tm (Figures 5A,B) and (ii) nuclear enriched Hog1 (Hog1-NLS-GFP) exhibited abrogated expression of Hog1-dependent ER stress-responsive genes, GPD2 and RHR2 (Figure 5D). It is plausible that Hog1-dependent cytosolic targets may migrate to the nucleus for activating ER stress-responsive transcriptional program. Contrary to this observation, Hogl does not depend on nuclear localization for expression of osmoresponsive genes in $C$. albicans, challenging the essentiality of nuclear translocation for inducing Hog1-dependent gene expression in response to all stresses (Day et al., 2017). Moreover, during ER stress, the cells expressing Hog1-CaaX-GFP retained phosphorylation for longer duration (Figure 5C), similar to osmotic stress conditions but in contrast to oxidative stress signal (Day et al., 2017). Dephosphorylation of Hog1 in S. cerevisiae is ascribed to the nuclear localized tyrosine phosphatase Ptp2 (ScPtp2) (Wurgler-Murphy et al., 1997). Likewise, the sustained phosphorylation of Hog1-GFP-CaaX can be attributed to its inaccessibility to the presumably nuclear localized phosphatases Ptp2 and Ptp3 (orthologs of ScPtp2) (Su et al., 2013). Our findings reaffirm the existence of stress-specific unique mechanisms that differentially affect the activation and cellular localization of Hog1, thus imparting specificity to the downstream outcomes in C. albicans.

Interestingly, a functional Hog1 is sufficient to promote adaptation to ER stress as both cytosolic as well as nuclear localized Hog1 was able to terminate Ire1-dependent UPR independently (Figure 6A). We speculate that Hog1 may be responding to ER stress in a biphasic manner, where the modification of preexisting cytosolic proteins may initiate the desensitization and termination of Ire1-dependent UPR, followed by the induction of Hog1-dependent nuclear genes required for the termination. Thus, this MAPK by virtue of its cytosol-dependent activity provides a fast and direct stress response that facilitates rapid relief from ER stress. Analogous to this observation is the role of Hog1 in regulating osmostress in a sequential manner by influencing the activity of a plasma membrane localized ion transporter during the early stages and subsequent activation of gene expression in $S$. cerevisiae (Proft and Struhl, 2004). Our results show a role of Hog1 in modulating the basal level (uninduced) of glycerol production (Figures 4B,C), a process that may be dependent on the cytosolic activity of Hog1. We show that absence of functional Hog1 is sufficient to reduce the basal glycerol content by 3fold, compared to wild type (Figure 4C), pointing to a link between the cytosolic activity of Hogl and glycerol production in C. albicans. The nautral osmolyte glycerol is known to shift the folding equilibrium toward the native conformation of the proteins without causing disturbances in other cellular processes, a strategy that allows cells to survive stress created by accumulation of misfolded proteins (Kumar, 2009). Thus, we propose that one of the cytosol-dependent physiological roles of Hogl could be to induce glycerol production in an attempt to survive ER stress conditions in C. albicans. Consistent with this, the role of cytosolic Hog1 in activating the upper branch of the glycolytic pathway, resulting in increased glycerol production during osmotic and ER stress conditions, has been established in S. cerevisiae (Torres-Quiroz et al., 2010). However, further work is required to confirm the cytosolic role of C. albicans Hog1 for glycerol production during ER stress. Thus, in an attempt to adapt to ER stress and avert cell death, C. albicans not only relies on Hacl-dependent structured activation but also on Hog1-dependent organized termination of the canonical Ire1dependent UPR. Additionally, through this study, the relevance of HOG pathway in fungal pathogenicity can be expanded also to its role in ER stress resistance. The finding that inactivation of HOG MAPK pathway can lock the Ire1-dependent UPR in an activated state for extended duration provides a basis for 
augmenting cell death in C. albicans, an additional vulnerable point for therapeutic intervention.

\section{DATA AVAILABILITY STATEMENT}

The original contributions presented in the study are included in the article/Supplementary Material, further inquiries can be directed to the corresponding author.

\section{AUTHOR CONTRIBUTIONS}

SP and FH provided the concept and design of the study. FH, PP, and ER performed the experiments and analyzed the data. SP wrote the manuscript. JP and ER reviewed the first draft of the manuscript. All authors approved the submitted version.

\section{FUNDING}

Work in the SLP laboratory was funded by an Umbrella grant awarded to Jawaharlal Nehru University under the scheme "Universities with Potential for Excellence (UPOE)". Additional funding from the SERB, Department of Science and Technology,

\section{REFERENCES}

Achkar, J. M., and Fries, B. C. (2010). Candida infections of the genitourinary tract. Clin. Microbiol. Rev. 23, 253-273. doi: 10.1128/CMR.00076-79

Albertyn, J., Hohmann, S., Thevelein, J. M., and Prior, B. A. (1994). GPD1, which encodes glycerol-3-phosphate dehydrogenase, is essential for growth under osmotic stress in Saccharomyces cerevisiae, and its expression is regulated by the high-osmolarity glycerol response pathway. Mol. Cell. Biol. 14, 4135-4144. doi: 10.1128/mcb.14.6.4135

Alonso-Monge, R., Navarro-García, F., Molero, G., Diez-Orejas, R., Gustin, M., Pla, J., et al. (1999). Role of the mitogen-activated protein kinase Hoglp in morphogenesis and virulence of Candida albicans. J. Bacteriol. 181, 3058-3068. doi: 10.1128/JB.181.10.3058-3068.1999

Alonso-Monge, R., Navarro-García, F., Román, E., Negredo, A. I., Eisman, B., Nombela, C., et al. (2003). The Hogl mitogen-activated protein kinase is essential in the oxidative stress response and chlamydospore formation in Candida albicans. Eukaryot. Cell 2, 351-361. doi: 10.1128/EC.2.2.351-361. 2003

Alonso-Monge, R., Román, E., Nombela, C., and Pla, J. (2006). The MAP kinase signal transduction network in Candida albicans. Microbiology 152, 905-912. doi: 10.1099/mic.0.28616-28610

Arana, D. M., Alonso-Monge, R., Du, C., Calderone, R., and Pla, J. (2007). Differential susceptibility of mitogen-activated protein kinase pathway mutants to oxidative-mediated killing by phagocytes in the fungal pathogen Candida albicans. Cell Microbiol. 9, 1647-1659. doi: 10.1111/j.1462-5822.2007.00898.x

Arana, D. M., Nombela, C., Alonso-Monge, R., and Pla, J. (2005). The Pbs2 MAP kinase kinase is essential for the oxidative-stress response in the fungal pathogen Candida albicans. Microbiology 151, 1033-1049. doi: 10.1099/mic.0. 27723-27720

Bicknell, A. A., Tourtellotte, J., and Niwa, M. (2010). Late phase of the endoplasmic reticulum stress response pathway is regulated by Hog1 MAP kinase. J. Biol. Chem. 285, 17545-17555. doi: 10.1074/jbc.M109.084681

Bongomin, F., Gago, S., Oladele, R., and Denning, D. (2017). Global and multinational prevalence of fungal diseases-estimate precision. J. Fungi 3:57. doi: 10.3390/jof3040057

Calderone, R. A., and Clancy, C. J. (eds) (2012). Candida and Candidiasis, 2nd Edn. Washington, DC: ASM Press.
Government of India under the Umbrella grant DST-PURSE, as well as UGC-Resource Networking, UGC-SAP, and DST-FIST is also acknowledged. Work in the JP laboratory was supported by Grants PGC2018-095047-B-I00 from MINECO and InGEMICS (B2017/BMD-3691) from CAM.

\section{ACKNOWLEDGMENTS}

We thank Janet Quinn for providing strains used in the study. We thank the current and the ex-members, especially Shabnam Sircaik, of the Panwar laboratory for critical reading of the manuscript. FH acknowledges University Grant Commission for Non-NET, and for awarding Junior and Senior Research Fellowships. PP acknowledges University Grant Commission for awarding Junior and Senior Research Fellowships and Indian Council of Medical Research for awarding Senior Research Fellowship.

\section{SUPPLEMENTARY MATERIAL}

The Supplementary Material for this article can be found online at: https://www.frontiersin.org/articles/10.3389/fmicb. 2021.794855/full\#supplementary-material

Chang, T.-K., Lawrence, D. A., Lu, M., Tan, J., Harnoss, J. M., Marsters, S. A., et al. (2018). Coordination between two branches of the unfolded protein response determines apoptotic cell fate. Mol. Cell 71, 629-636.e5. doi: 10.1016/j.molcel. 2018.06.038.

Chauhan, N., Inglis, D., Roman, E., Pla, J., Li, D., Calera, J. A., et al. (2003). Candida albicans response regulator gene SSK1 regulates a subset of genes whose functions are associated with cell wall biosynthesis and adaptation to oxidative stress. Eukaryot. Cell 2, 1018-1024.

Chawla, A., Chakrabarti, S., Ghosh, G., and Niwa, M. (2011). Attenuation of yeast UPR is essential for survival and is mediated by IRE1 kinase. J. Cell Biol. 193, 41-50. doi: 10.1083/jcb.201008071

Cheetham, J., MacCallum, D. M., Doris, K. S., da Silva, Dantas, A., Scorfield, S., et al. (2011). MAPKKK-independent regulation of the Hog1 stress-activated protein kinase in Candida albicans. J. Biol. Chem. 286, 42002-42016. doi: 10. 1074/jbc.M111.265231

Cheetham, J., Smith, D. A., da Silva, Dantas, A., Doris, K. S., Patterson, M. J., et al. (2007). A single MAPKKK regulates the Hogl MAPK pathway in the pathogenic fungus Candida albicans. Mol. Biol. Cell 18, 4603-4614. doi: 10. 1091/mbc.e07-06-0581

Cox, J. S., Shamu, C. E., and Walter, P. (1993). Transcriptional induction of genes encoding endoplasmic reticulum resident proteins requires a transmembrane protein kinase. Cell 73, 1197-1206.

Day, A. M., Herrero-de-Dios, C. M., MacCallum, D. M., Brown, A. J. P., and Quinn, J. (2017). Stress-induced nuclear accumulation is dispensable for Hog1dependent gene expression and virulence in a fungal pathogen. Sci. Rep. 7:14340. doi: 10.1038/s41598-017-14756-14754

Edmond, M. B., Wallace, S. E., McClish, D. K., Pfaller, M. A., Jones, R. N., and Wenzel, R. P. (1999). Nosocomial bloodstream infections in United States hospitals: a three year analysis. Clin. Infect. Dis. 29, 239-244. doi: 10.1086/ 520192

Eisman, B., Alonso-Monge, R., Román, E., Arana, D., Nombela, C., and Pla, J. (2006). The Cek1 and Hogl mitogen-activated protein kinases play complementary roles in cell wall biogenesis and chlamydospore formation in the fungal pathogen Candida albicans. Eukaryot. Cell 5, 347-358. doi: 10.1128/ EC.5.2.347-358.2006

Enjalbert, B., Smith, D. A., Cornell, M. J., Alam, I., Nicholls, S., Brown, A. J. P., et al. (2006). Role of the Hog1 stress-activated protein kinase in the global 
transcriptional response to stress in the fungal pathogen Candida albicans. Mol. Biol. Cell 17, 1018-1032. doi: 10.1091/mbc.e05-06-0501

Ghannoum, M. A., Jurevic, R. J., Mukherjee, P. K., Cui, F., Sikaroodi, M., Naqvi, A., et al. (2010). Characterization of the oral fungal microbiome (mycobiome) in healthy individuals. PLoS Pathog 6:e1000713. doi: 10.1371/journal.ppat. 1000713

Gillum, A. M., Tsay, E. Y. H., and Kirsch, D. R. (1984). Isolation of the Candida albicans gene for orotidine-5'-phosphate decarboxylase by complementation of S. cerevisiae ura3 and E. coli pyrF mutations. Mol. Gen. Genet. 198, 179-182. doi: 10.1007/BF00328721

Guerro-Moreno, A., Ang, J., Welsch, H., Jochem, M., and Hanna, J. (2019). Regulation of the unfolded protein response in yeast by oxidative stress. FEBS Lett. 593, 1080-1088. doi: 10.1002/1873-3468.13389

Hohmann, S. (2002). Osmotic stress signaling and osmoadaptation in yeasts. Microbiol. Mol. Biol. Rev. 66, 300-372.

Hopke, A., Nicke, N., Hidu, E. E., Degani, G., Popolo, L., and Wheeler, R. T. (2016). Neutrophil attack triggers extracellular trap-dependent Candida cell wall remodeling and altered immune recognition. PLoS Pathog 12:e1005644. doi: 10.1371/journal.ppat.1005644

Kaloriti, D., Jacobsen, M., Yin, Z., Patterson, M., Tillmann, A., Smith, D. A., et al. (2014). Mechanisms underlying the exquisite sensitivity of Candida albicans to combinatorial cationic and oxidative stress that enhances the potent fungicidal activity of phagocytes. mBio 5:e1334-14. doi: 10.1128/mBio.01334-1314

Kan, S., Pang, Q., Song, N., Mei, H., Zheng, H., Li, D., et al. (2020). Study on Vulvovaginal Candidiasis: Clinical Epidemiology and in Vitro Susceptibility of Pathogenic Yeasts in China. Rochester, NY: SSRN.

Kawahara, T., Yanagi, H., Yura, T., and Mori, K. (1997). Endoplasmic reticulum stress-induced mRNA splicing permits synthesis of transcription factor Hac1p/Ern4p that activates the unfolded protein response. Mol. Biol. Cell 8, 1845-1862. doi: $10.1091 / \mathrm{mbc} .8 \cdot 10.1845$

Kennedy, M. J., and Volz, P. A. (1985). Effect of various antibiotics on gastrointestinal colonization and dissemination by Candida albicans. Sabouraudia 23, 265-273. doi: 10.1080/00362178585380391

Kohno, K., Normington, K., Sambrook, J., Gething, M. J., and Mori, K. (1993). The promoter region of the yeast KAR2 (BiP) gene contains a regulatory domain that responds to the presence of unfolded proteins in the endoplasmic reticulum. Mol. Cell. Biol. 13, 877-890.

Korennykh, A. V., Egea, P. F., Korostelev, A. A., Finer-Moore, J., Zhang, C., Shokat, K. M., et al. (2009). The unfolded protein response signals through high-order assembly of Ire1. Nature 457, 687-693. doi: 10.1038/nature07661

Krishnan, K., and Askew, D. S. (2014). Endoplasmic reticulum stress and fungal pathogenesis. Fungal Biol. Rev. 28, 29-35. doi: 10.1016/j.fbr.2014.07.001

Krysan, D. J. (2009). The cell wall and endoplasmic reticulum stress responses are coordinately regulated in Saccharomyces cerevisiae. Commun. Integr. Biol. 2, 233-235.

Kumamoto, C. A. (2011). Inflammation and gastrointestinal Candida colonization. Curr. Opin. Microbiol. 14, 386-391. doi: 10.1016/j.mib.2011.07.015

Kumar, R. (2009). Role of naturally occurring osmolytes in protein folding and stability. Arch. Biochem. Biophys. 491, 1-6. doi: 10.1016/j.abb.2009.09.007

Mayer, F. L., Wilson, D., and Hube, B. (2013). Candida albicans pathogenicity mechanisms. Virulence 4, 119-128. doi: 10.4161/viru.22913

Mizuno, T., Masuda, Y., and Irie, K. (2015). The Saccharomyces cerevisiae AMPK, Snf1, negatively regulates the Hog1 MAPK pathway in ER stress response. PLoS Genet. 11:e1005491. doi: 10.1371/journal.pgen.1005491

Mori, K., Ma, W., Gething, M. J., and Sambrook, J. (1993). A transmembrane protein with a cdc2+/CDC28-related kinase activity is required for signaling from the ER to the nucleus. Cell 74, 743-756. doi: 10.1016/0092-8674(93) 90521-q

Munro, C. A., Selvaggini, S., de Bruijn, I., Walker, L., Lenardon, M. D., Gerssen, B., et al. (2007). The PKC, $\mathrm{HOG}$ and $\mathrm{Ca}^{2+}$ signalling pathways co-ordinately regulate chitin synthesis in Candida albicans. Mol. Microbiol. 63, 1399-1413. doi: 10.1111/j.1365-2958.2007.05588.x

Negredo, A., Monteoliva, L., Gil, C., Pla, J., and Nombela, C. Y. (1997). Cloning, analysis and one-step disruption of the ARG5,6 gene of Candida albicans. Microbiology 143, 297-302. doi: 10.1099/00221287-143-2-297

Perlroth, J., Choi, B., and Spellberg, B. (2007). Nosocomial fungal infections: epidemiology, diagnosis, and treatment. Med. Mycol. 45, 321-346. doi: 10.1080/ 13693780701218689
Petelenz-Kurdziel, E., Kuehn, C., Nordlander, B., Klein, D., Hong, K.-K., Jacobson, T., et al. (2013). Quantitative analysis of glycerol accumulation, glycolysis and growth under hyper osmotic stress. PLoS Comput. Biol. 9:e1003084. doi: 10. 1371/journal.pcbi.1003084

Prieto, D., Román, E., Correia, I., and Pla, J. (2014). The HOG pathway is critical for the colonization of the mouse gastrointestinal tract by Candida albicans. PLoS One 9:e87128. doi: 10.1371/journal.pone.0087128

Proft, M., and Struhl, K. (2004). MAP kinase-mediated stress relief that precedes and regulates the timing of transcriptional induction. Cell 118, 351-361. doi: 10.1016/j.cell.2004.07.016

Román, E., Correia, I., Prieto, D., Alonso, R., and Pla, J. (2020). The HOG MAPK pathway in Candida albicans: more than an osmosensing pathway. Int. Microbiol. 23, 23-29. doi: 10.1007/s10123-019-00069-61

Ruggiano, A., Foresti, O., and Carvalho, P. (2014). ER-associated degradation: protein quality control and beyond. J. Cell Biol. 204, 869-879. doi: 10.1083/jcb. 201312042

San José, C., Monge, R. A., Pérez-Díaz, R., Pla, J., and Nombela, C. (1996). The mitogen-activated protein kinase homolog HOG1 gene controls glycerol accumulation in the pathogenic fungus Candida albicans. J. Bacteriol. 178, 5850-5852.

Sircaik, S., Román, E., Bapat, P., Lee, K. K., Andes, D. R., Gow, N. A. R., et al. (2021). The protein kinase Irel impacts pathogenicity of Candida albicans by regulating homeostatic adaptation to endoplasmic reticulum stress. Cell Microbiol. 23:e13307. doi: 10.1111/cmi.13307

Smith, D. A., Nicholls, S., Morgan, B. A., Brown, A. J. P., and Quinn, J. (2004). A conserved stress-activated protein kinase regulates a core stress response in the human pathogen Candida albicans. Mol. Biol. Cell 15, 4179-4190. doi: 10.1091/mbc.e04-03-0181

Su, C., Lu, Y., and Liu, H. (2013). Reduced TOR signaling sustains hyphal development in Candida albicans by lowering Hog1 basal activity. Mol. Biol. Cell 24, 385-397. doi: 10.1091/mbc.e12-06-0477

Thomas, E., Sircaik, S., Roman, E., Brunel, J.-M., Johri, A. K., Pla, J., et al. (2015). The activity of RTA2, a downstream effector of the calcineurin pathway, is required during tunicamycin-induced ER stress response in Candida albicans. FEMS Yeast Res. 15:fov095. doi: 10.1093/femsyr/fov095

Torres-Quiroz, F., García-Marqués, S., Coria, R., Randez-Gil, F., and Prieto, J. A. (2010). The activity of yeast Hog1 MAPK is required during endoplasmic reticulum stress induced by tunicamycin exposure. J. Biol. Chem. 285, 2008820096. doi: 10.1074/jbc.M109.063578

Wimalasena, T. T., Enjalbert, B., Guillemette, T., Plumridge, A., Budge, S., Yin, Z., et al. (2008). Impact of the unfolded protein response upon genome-wide expression patterns, and the role of $\mathrm{Hacl}$ in the polarized growth, of Candida albicans. Fungal Genet. Biol. 45, 1235-1247. doi: 10.1016/j.fgb.2008.06.001

Woolford, C. A., Lagree, K., Xu, W., Aleynikov, T., Adhikari, H., Sanchez, H., et al. (2016). bypass of Candida albicans filamentation/biofilm regulators through diminished expression of protein kinase Cak1. PLoS Genet. 12:e1006487. doi: 10.1371/journal.pgen.1006487

Wurgler-Murphy, S. M., Maeda, T., Witten, E. A., and Saito, H. (1997). Regulation of the Saccharomyces cerevisiae HOG1 mitogen-activated protein kinase by the PTP2 and PTP3 protein tyrosine phosphatases. Mol. Cell Biol. 17, 1289-1297.

Conflict of Interest: The authors declare that the research was conducted in the absence of any commercial or financial relationships that could be construed as a potential conflict of interest.

Publisher's Note: All claims expressed in this article are solely those of the authors and do not necessarily represent those of their affiliated organizations, or those of the publisher, the editors and the reviewers. Any product that may be evaluated in this article, or claim that may be made by its manufacturer, is not guaranteed or endorsed by the publisher.

Copyright (c) 2022 Husain, Pathak, Román, Pla and Panwar. This is an open-access article distributed under the terms of the Creative Commons Attribution License (CC BY). The use, distribution or reproduction in other forums is permitted, provided the original author(s) and the copyright owner(s) are credited and that the original publication in this journal is cited, in accordance with accepted academic practice. No use, distribution or reproduction is permitted which does not comply with these terms. 